\title{
Origin of artificial electrodynamics in three-dimensional bosonic models
}

\author{
O. I. Motrunich \\ Kavli Institute for Theoretical Physics, University of California, Santa Barbara, California 93106-4030, USA \\ T. Senthil \\ Massachusetts Institute of Technology, 77 Massachusetts Avenue, Cambridge, Massachusetts 02139-4307, USA
}

(Received 10 August 2004; published 3 March 2005)

\begin{abstract}
Several simple models of strongly correlated bosons on three-dimensional lattices have been shown to possess exotic fractionalized Mott insulating phases with a gapless "photon" excitation. In this paper we show how to view the physics of this "Coulomb" state in terms of the excitations of proximate superfluid. We argue for the presence of ordered vortex cores with a broken discrete symmetry in the nearby superfluid phase and that proliferating these degenerate but distinct vortices with equal amplitudes produces the Coulomb phase. This provides a simple physical description of the origin of the exotic excitations of the Coulomb state. The physical picture is formalized by means of a dual description of three-dimensional bosonic systems in terms of fluctuating quantum mechanical vortex loops. Such a dual formulation is extensively developed. It is shown how the Coulomb phase (as well as various other familiar phases) of three-dimensional bosonic systems may be described in this vortex loop theory. For bosons at half-filling and the closely related system of spin-1/2 quantum magnets on a cubic lattice, fractionalized phases as well as bond- or "box"-ordered states are possible. The latter are analyzed by an extension of techniques previously developed in two spatial dimensions. The relation between these "confining" phases with broken translational symmetry and the fractionalized Coulomb phase is exposed.
\end{abstract}

DOI: 10.1103/PhysRevB.71.125102

PACS number(s): 71.10.Hf, 74.20.Mn

\section{INTRODUCTION}

Several recent studies have produced a variety of models that exhibit quantum number fractionalization in two dimensions (2D) and in the absence of magnetic fields. ${ }^{1-5}$ It has been appreciated for some time that similar fractionalization phenomenon can occur also in three dimensions and can take novel forms not possible in $2 \mathrm{D} .^{5-9}$ In particular, so-called Coulomb phases have recently been demonstrated in 3D bosonic models. In these Coulomb phases the fractionalized degrees of freedom interact with a gapless emergent $U(1)$ gauge field. The gauge excitations describe, at low energies and long distances, a linear dispersing transverse mode which has been dubbed an "artificial photon." The adjective "artificial" refers to the fact that this photon really corresponds to some collective excitation of the boson system and has nothing directly to do with the true electromagnetic photon. In particular the artificial photon in the fractionalized 3D Coulomb phases arises even in microscopic models of correlated bosons with purely short-ranged interactions. The Coulomb phase also contains additional gapped excitations that may be identified with magnetic monopoles of the internal gauge field. The possibility of such dynamical generation of "light" was noted more than two decades ago in Ref. 10.

In the case of fractionalization in $2 \mathrm{D}$ bosonic systems, a very fruitful perspective is obtained by departing from a superfluid state and considering nearby Mott insulator phases as vortex condensates. ${ }^{11-14}$ Conventional insulating states of bosons, with possibly charge or bond order, can be understood in these terms. The same perspective also naturally predicts occurrence of a $Z_{2}$ fractionalized state when the superfluidity is destroyed by the condensation of doubled vor- tices while single ones remain gapped. The unpaired single vortex survives in the resulting insulating phase as a gapped excitation that retains a $Z_{2}$ quantum number (only the oddity of vorticity remains well defined) - this is the $Z_{2}$ vortex or vison excitation of this fractionalized insulator.

Turning to $3 \mathrm{D}$ systems, we envisage a similar perspective on insulating states as vortex condensates, except that vortex excitations in the superfluid are now lines instead of point particles. For example, a $Z_{2}$ fractionalized insulator is obtained by destroying the superfluid order by pairing and condensing vortex lines.

The goal of the present paper is to develop a similar intuition for the 3D Coulomb phase [also dubbed the $U(1)$ phase]. In particular, we want to understand the genesis of the excitation structure of this phase in an approach that departs from the superfluid state. We note that the excitation structure of the Coulomb phase consists of gapped charge$1 / 2$ chargons that are minimally coupled to an emergent gapless "photon" mode. In addition, there is another gapped excitation that may be viewed as a "magnetic monopole" that acts as a source of the emergent magnetic flux. The picture that appears from the present study is that physics inside vortex cores is intimately involved in producing the $U(1)$ phase. We thus have a rather unusual but very interesting situation when we cannot ignore the microscopics of the vortex core when describing the relationship of the superfluid phase to the nearby insulating states.

The main idea is very simply illustrated by considering bosons on a three-dimensional cubic lattice and at an average filling of one-half boson per site. In some semiclassical descriptions, there are two simple phases that might be imagined for the bosons. First, there is a translationally invariant 

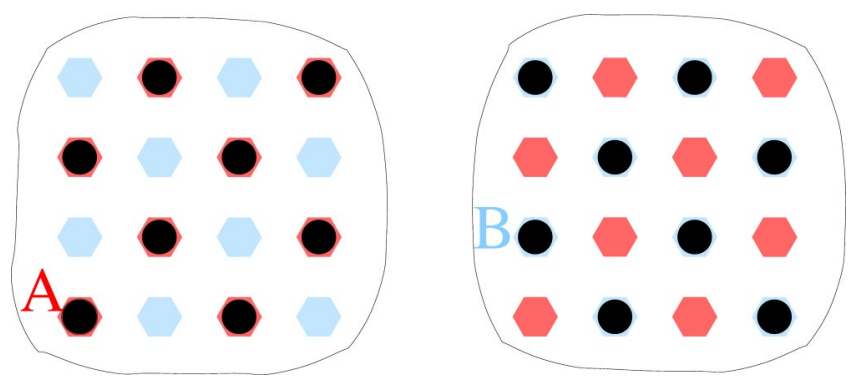

FIG. 1. View of the cross section of a vortex line in a threedimensional superfluid of bosons at half-filling on a cubic lattice. Two checkerboard ordering patterns-where the boson density is higher on one of the two sublattices - are possible inside the vortex core.

superfluid phase. Second, there is a Mott insulator in which the bosons preferentially occupy sites on one or the other of the two sublattices. Clearly, such a Mott insulating ground state is twofold degenerate corresponding to the two possible states of boson charge ordering. The superfluid phase possesses vortices which are extended line defects. The superfluid order parameter will be suppressed in the core of such a vortex line. If the vortex core size is larger than the lattice spacing (as might well happen), it makes sense to ask about what "phase" is obtained in the core. An immediate guess is that the core is simply the checkerboard charge-ordered Mott insulating state as illustrated in Fig. 1. However, the checkerboard state breaks a symmetry (that of sublattice interchange) and is twofold degenerate. We are then led to conclude that there will be two kinds of vortices depending on which sublattice has higher boson density in the core.

This simpleminded discussion on the structure of vortices will be modified once we take quantum fluctuations into account more seriously in the vortex core. In particular, as the core has finite radius, it essentially behaves as a onedimensional system. Quantum fluctuations will tend to restore the broken symmetry in the core and produce a unique vortex line. However, as the broken sublattice symmetry is discrete, it is natural to expect that the symmetry breaking will be stable to weak quantum fluctuations. Thus there will be some range of parameters in which there will be two distinct kinds of vortex lines with symmetry-broken cores. In other range of parameters a unique vortex with no symmetry breaking in the core may well result. The change between the two parameter ranges involves no ground-state phase transition but only a change in the nature of the excited states.

Now consider disordering the superfluid by proliferating and condensing vortex line loops. Clearly, the insulating phase that obtains will depend on the structure of the vortices that are actually proliferating. Consider the situation where the vortices have ordered cores. If we preferentially condense one or the other kind of vortex, we will clearly induce checkerboard charge order in the insulator. But what if we condense both species of vortices with equal amplitude? We argue that the resulting phase is the Coulomb fractionalized insulator. We show how the excitations of this exotic insulator may be understood in terms of the various excitations of the superfluid when it has ordered vortex cores.

Consider a vortex line. It is still possible for the core order to change from one state to the other somewhere along the
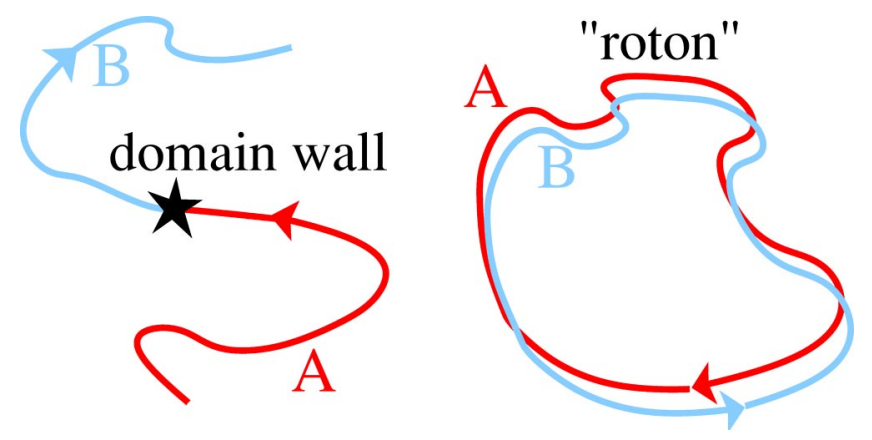

FIG. 2. Vortex lines with different core orders are indicated as $A$ and $B$. Left panel: a domain wall associated with the order in the core. If the superfluid is disordered by proliferating both vortex species simultaneously while keeping such domain walls gapped, the Coulomb phase results. The gapped domain walls survive as the monopoles of the Coulomb phase. Right panel: a "roton" loop is formed by bringing together a vortex with one ordered core and an antivortex with the other core. These particular roton loops correspond to loops of emergent magnetic flux which are strongly fluctuating in the Coulomb phase. The artificial photon is associated with fluctuations of these loops.

line as shown in Fig. 2, but there is a gap for such a domain wall excitation. We expect this gap to remain when such vortices proliferate while retaining their order. When both vortex species proliferate, the domain wall excitations become point particles which we identify with the monopoles of the Coulomb phase.

The appearance of the artificial photon is more subtle, but is also a consequence of proliferating two species of vortices. To explain this, it is useful to recall what happens in the more familiar situation of particle condensates. When the particle that is condensing is "neutral" (in the sense of having only short ranged interactions), the resulting condensate supports gapless Goldstone excitations. On the other hand, if it is charged (in the sense of having long-ranged interactions), there are no gapless excitations associated with the condensation (the Anderson-Higgs phenomenon). Consider now the dual description of bosonic Mott insulators in three spatial dimensions as "condensates" of extended vortex loops. For such a line condensate we may again expect that if the vortices have long-ranged interactions, then the resulting condensate supports only gapped excitations. However, consider now the situation envisaged above where there are two vortex species (distinguished by the ordering in the core) which both condense. We may form a "roton" line from these two species by bringing together a vortex with one ordered core and an antivortex with the other core, and this is pictured in Fig. 2. Such objects have no net vorticity, and therefore only short-ranged interactions with one another. When both vortex species condense such roton loops condense as well. By analogy to particle condensates we may expect then to find gapless excitations. We will show in this paper that this is indeed the case and that the resulting gapless modes may be identified with the photon of the Coulomb phase. More precisely, we will argue that the roton lines described above may be viewed as the magnetic field lines of the emergent deconfined $U(1)$ gauge field that obtains in the Coulomb phase. 
It is useful to point a connection with a recent study ${ }^{15}$ of so-called deconfined critical points in two dimensions such as separating a superfluid and a bond density-wave insulator of bosons at half-filling on a square lattice. In the superfluid phase, we can speak of two vortex species with checkerboard charge order in the core (vortices are now point particles). There is a high-order mixing of the two species involving four vortices of one type changing simultaneously to the other type, so the vortex identity potentially becomes problematic as the gap to the vortex excitations is decreased. The result of Ref. 15 is that the tunneling that mixes the two species is in fact dangerously irrelevant at the transition to the bond density-wave state, and the long-wavelength description contains two vortex fields with a separate number conservation for each species. The tunneling is relevant when the vortices condense and produces an insulating state with specific bond order. As elaborated in Ref. 15 there is a large intermediate region of length scales in the insulator (close to the critical point) which may be described as a two-dimensional version of the Coulomb phase. The (intermediate) long-wavelength physics of this region is described in terms of a gapless excitation which may be regarded as the two-dimensional version of the photon; this "photon" becomes massive at longer wavelengths because of the proliferation of the discussed tunneling events. In contrast, in three dimensions, condensing vortices with ordered cores is a stable possibility at all length scales and gives the Coulomb phase at the focus of the present study.

The primary purpose of this paper is to develop the physical picture sketched above for the excitations of the Coulomb phase in some detail. This goal is strongly aided by a formal dual description of three-dimensional bosonic systems in terms of extended fluctuating vortex loops. This duality transformation-a generalization to $(3+1) \mathrm{D}$ of the familiar boson-vortex duality in two spatial dimensions-has been available in the literature for many years, and is reviewed in Sec. IV. Here we will use this and further develop it extensively to obtain a dual description of the phases of various three-dimensional bosonic systems which gives substance to the physical pictures. In the course of these studies, we develop a description of bosons at half-filling on a threedimensional cubic lattice that is capable of describing all the insulating phases discussed above- the Coulomb phase, the checkerboard charge ordered phase, and also different bond density-wave ordered phases. This gives a three-dimensional generalization of the corresponding development in Ref. 15. Boson systems at half-filling are closely related to spin- $1 / 2$ quantum magnets. Thus some of our results have direct implications for quantum spin systems on cubic lattices as well. Indeed we will borrow from techniques familiar from studies of quantum antiferromagnets in two spatial dimensions and generalize them in discussing the structure of the bondordered insulating phases of bosons at half-filling

The paper is organized as follows. In Sec. II, we first review a simple bosonic model that explicitly realizes the fractionalized Coulomb phase. This provides a good starting point for the discussion of the relationship of the $U(1)$ state to other phases. The model has integer boson filling and is simple to analyze in the charge representation, but the vortex physics is obscured by the nontrivial lattice structure. Still,

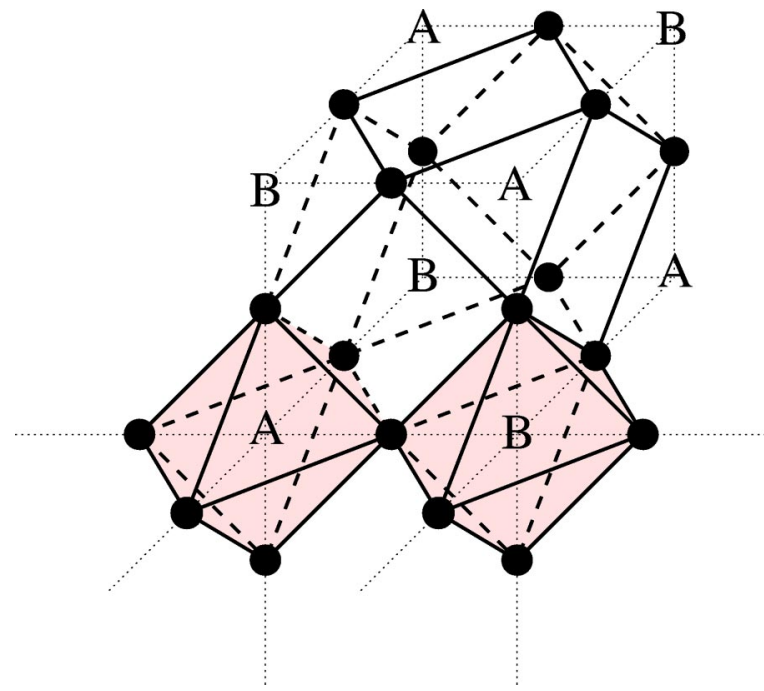

FIG. 3. Explicit model that realizes the Coulomb phase. Boson islands are located on the link-centered sites of a simple cubic lattice. The islands can be also viewed as forming a network of cornersharing octahedra, and two octahedron units are shown. In the Hamiltonian, Eq. (1), we stipulate a term $U N_{r}^{2}$ which prefers charge neutrality of each octahedron.

the model motivates an effective theory, formulated as a compact $U(1)$ gauge theory with two matter fields (chargons), that properly captures the possible phases and is amenable to an analytic description in terms of vortices, furnishing the main argument for the proposed physical picture. In Sec. III, we consider vortex phenomenology in this twochargon theory. Precise duality analysis is presented in Sec. $\mathrm{IV}$, where we first review the conventional boson-vortex duality in $(3+1) \mathrm{D}$ from a modern perspective and then describe how to include the physics of two vortex species in the dual description (the details of the derivation are given in Appendix A). In Appendix D, we consider a (2+1)D Ising version of the specific boson model in order to give some intuition in a more simple setting; this toy model realizes a topological $Z_{2}$ phase in two dimensions via a mechanism that resembles the one proposed for the Coulomb phase in three dimensions, with the vortices replaced by Ising domain walls. In Sec. V and supporting Appendixes B and C, we develop a dual description for bosons at half-integer filling. An important ingredient of this description is monopole Berry phases generalizing Haldane's result to three-dimensional spin-1/2 quantum magnets on a cubic lattice. We apply this in Sec. VI, where we show how bond density-wave states can be analyzed as monopole condensates coming from the Coulomb phase.

\section{REALIZATION OF THE COULOMB PHASE AND PHENOMENOLOGY}

\section{A. Review of the boson Hubbard model}

We begin with a review of a specific model that realizes Coulomb phase in 3D. ${ }^{5,6,8}$ The model is formulated in terms of soft-core bosons (quantum rotors) residing on linkcentered sites ("islands") of a simple cubic lattice as depicted 


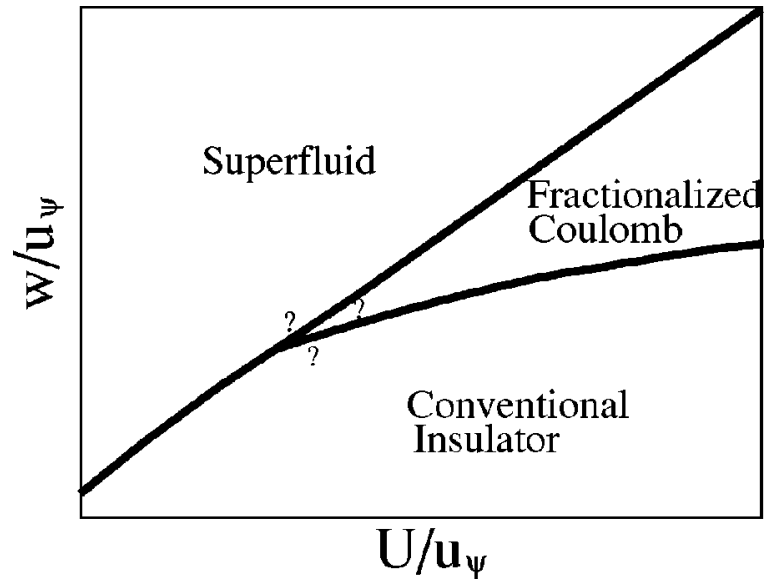

FIG. 4. Schematic phase diagram of the boson model, Eq. (1), exhibiting the stable phases discussed in the text; note that we do not know the details for intermediate-coupling strengths and whether some other states may intervene.

in Fig. 3. The boson islands can also be viewed as forming a lattice of corner-sharing octahedra. Bosons can hop with amplitude $w$ between neighboring islands. Besides the usual onsite repulsion $u_{\psi}$, we also include repulsive interactions between the bosons that favor charge neutrality of each octahedron. The complete Hamiltonian is

$$
H=-w \sum_{\left\langle l l^{\prime}\right\rangle}\left(\psi_{l}^{\dagger} \psi_{l^{\prime}}+\text { H.c. }\right)+u_{\psi} \sum_{l} n_{l}^{2}+U \sum_{r} N_{r}^{2},
$$

where $\psi_{l}^{\dagger}=e^{i \phi_{l}}$ creates a boson on a given island and $n_{l}$ is the corresponding boson number operator. Each octahedron is labeled by the cubic lattice site $r$ at its center; the corresponding operator $N_{r}$ is defined through

$$
N_{r}=\sum_{l \in r} n_{l},
$$

where $l \in r$ sums over all cubic lattice links emanating from $r$. The total boson number of the system is $N_{\text {tot }}=\frac{1}{2} \sum_{r} N_{r}$.

We summarize the analysis of the possible phases of the above Hamiltonian. When the boson hopping dominates, $w$ $\gg u_{\psi}, U$, the system is a superfluid. When the charging energy dominates, $u_{\psi}, U \gg w$, the system is a conventional Mott insulator. On the other hand, as reviewed below, when the charging energies $U$ and $u_{\psi}$ are varied separately, there is an intermediate regime $U \gg w \gg \sqrt{u_{\psi} U}$ such that the system is in a Coulomb phase. ${ }^{5}$ This phase is a fractionalized insulator with the excitation spectrum consisting of gapped charge$1 / 2$ chargons, a gapless linearly dispersing photon, and a gapped monopole. A schematic phase diagram of our model is shown in Fig. 4.

The analysis in the limit $U \gg w, u_{\psi}$ is similar to that in the large- $U$ limit of the electronic Hubbard model at half-filling. For $w=u_{\psi}=0$ there is a degenerate manifold of ground states specified by the requirement $N_{r}=0$ for each $r$, separated by a large charge gap $U$ from the nearest sectors. Including the $w$, $u_{\psi}$ terms lifts the degeneracy in each such sector, and this is best described by deriving the corresponding effective Hamiltonians for small perturbing couplings.
In the ground-state sector $N_{r}=0$ for all $r$, an elementary calculation gives

$$
H_{\text {eff }}^{(0)}=u_{\psi} \sum_{l} n_{l}^{2}-K_{\text {ring }} \sum_{\square}\left(\psi_{12}^{\dagger} \psi_{23} \psi_{34}^{\dagger} \psi_{41}+\text { H.c. }\right),
$$

with $K_{\text {ring }}=2 w^{2} / U$. When writing the "ring exchange" term around a given placket, we label a boson island by the corresponding link end points.

A simple change of variables shows that $H_{\text {eff }}^{(0)}$ together with the constraint $N_{r}=0$ can be regarded as the well-studied $(3+1) \mathrm{D}$ compact $U(1)$ gauge theory. Indeed, divide the underlying cubic lattice of Fig. 3 into $A$ and $B$ sublattices. We now define a vector field $a_{r \alpha} \equiv \eta_{r} \phi_{r, r+\alpha}$, where $\eta_{r}=+1$ if $r$ $\in A$ and $\eta_{r}=-1$ if $r \in B, \alpha=\hat{x}, \hat{y}, \hat{z}$; we also perform the corresponding transformation to the vector field $E_{r \alpha} \equiv \eta_{r} n_{r, r+\alpha}$ conjugate to $a_{r \alpha}$. In the new variables,

$$
N_{r}=\eta_{r} \boldsymbol{\nabla} \cdot \boldsymbol{E}
$$

If $a_{r \alpha}$ is interpreted as a compact $U(1)$ vector potential and $E_{r \alpha}$ as the corresponding electric field operator, the groundstate sector constraint $N_{r}=0$ is simply the Gauss law, while the effective Hamiltonian has the declared lattice gauge theory form

$$
H_{\text {eff }}^{(0)}=u_{\psi} \sum_{r, \alpha} E_{r \alpha}^{2}-2 K_{\text {ring }} \sum \cos (\nabla \times \boldsymbol{a}) .
$$

In $(3+1) \mathrm{D}$, the compact $U(1)$ gauge theory has two distinct phases: For $K_{\text {ring }} \lesssim u_{\psi}$, the gauge theory is confining, and all excitations carrying nonzero gauge charge are confined. Zero gauge charge excitations of course exist with a gap $\propto U$; these carry integer physical charge in units of elementary boson charge $q_{b}$. This is the conventional Mott insulator of our boson model.

In the opposite regime $K_{\text {ring }} \gtrsim u_{\psi}$, the gauge theory is in the deconfined Coulomb phase and has a gapless linearly dispersing gauge boson (photon) and a gapped topological point defect (monopole) as its distinct excitations in the $N_{r}$ $=0$ sector. In the charged sectors, objects with $N_{r}=1$ at some site-i.e., physical charge $q_{b} / 2$-are not confined and can propagate above a finite gap of order $U$. These charged excitations interact via an emergent long-range Coulomb interaction. A detailed description of the ground-state properties of the Coulomb phase due to the gapless photon can be found in Ref. 8.

This completes the review part of our discussion, and we now focus on the relationship of the $U(1)$ phase to the nearby more conventional phases. A two-dimensional quantum Ising version of the corner-sharing octahedra model is considered in Appendix D.

\section{B. Phenomenological gauge theory description}

We want to obtain a description of the Coulomb phase that includes the chargon fields; this is required if we want to discuss the transition from the Coulomb phase to the superfluid as the charge gap collapses to zero. To this end, we need to consider charge-carrying excitations in more detail. In the large- $U$ limit, this is done by deriving effective Hamil- 
tonians in the charged sectors, just as we did in the groundstate sector, Eq. (2). For example, to study the motion of a single chargon in a given region, we consider the sector with $N_{r}=\delta_{r r_{0}}$ where $r_{0}$ can be anywhere in this region. By inspecting the possible moves of the chargon, we find that it can hop only on the sites of the same sublattice of the cubic lattice. More generally, the total chargon number on a given sublattice is conserved for any chargon motion that derives from the microscopic boson hopping. This means that there are two distinct chargons and can be also seen directly from the Gauss law, Eq. (3): An excitation with the gauge charge +1 carries boson charge $+q_{b} / 2$ if it resides on the $A$ sublattice and $-q_{b} / 2$ if it resides on the $B$ sublattice, and in the Coulomb phase the gauge and boson charges are both good quantum numbers.

Instead of working directly with the microscopically derived effective Hamiltonian for the chargons, which is complicated, we consider the following model gauge theory:

$$
\begin{aligned}
H_{2 c h}= & U \sum_{r}\left(n_{1 r}^{2}+n_{2 r}^{2}\right)-t \sum_{r r^{\prime}}\left(b_{1 r}^{\dagger} b_{1 r^{\prime}} e^{\left.i a_{r r^{\prime}}+b_{2 r}^{\dagger} b_{2 r^{\prime}} e^{i a_{r r^{\prime}}}\right)}\right. \\
& +u_{E} \sum \boldsymbol{E}^{2}-K \sum \cos (\boldsymbol{\nabla} \times \boldsymbol{a}) .
\end{aligned}
$$

This has two chargon fields minimally coupled to the compact $U(1)$ gauge field. The Hilbert space of the theory is defined by

$$
\boldsymbol{\nabla} \cdot \boldsymbol{E}=n_{1 r}+n_{2 r} .
$$

$b_{1 r}^{\dagger}$ and $b_{2 r}^{\dagger}$ both carry gauge charge +1 , and we take the first to carry boson charge $+q_{b} / 2$ and the second $-q_{b} / 2$. There is no discrimination between the two chargons, so the hopping amplitude and the on-site interaction are taken equal for the two species. This theory has the correct chargon content and is therefore expected to capture the relevant physics of the microscopic model.

For later convenience, we also write down the corresponding $(3+1) \mathrm{D}$ classical action

$$
\begin{aligned}
S_{2 c h}= & -\beta \sum\left[\cos \left(\nabla_{\mu} \phi_{1}-a_{\mu}\right)+\cos \left(\nabla_{\mu} \phi_{2}-a_{\mu}\right)\right] \\
& -K \sum \cos \left(\nabla_{\mu} a_{\nu}-\nabla_{\nu} a_{\mu}\right) .
\end{aligned}
$$

For simplicity, the action is written in a space-time isotropic form and is characterized by two coupling constants $\beta$ and $K$. The former characterizes the relative strength of the chargon hopping $t$ versus repulsion $U$, while the latter characterizes the competition of $K$ vs $u_{E}$.

As an aside, we note that the above gauge theory also appears in a particular "slave-boson" treatment of a generic bosonic Hamiltonian at integer filling:

$$
H_{\text {generic }}=u_{b} \sum_{r} n_{r}^{2}-w_{b} \sum_{r r^{\prime}} b_{r}^{\dagger} b_{r^{\prime}}+\cdots
$$

Specifically, consider an analog of $C P^{1}$ representation for the bosons treated as $O(2)$ rotors:

$$
b_{r}^{\dagger} \equiv e^{i \phi_{r}}=b_{1 r}^{\dagger} b_{2 r} \equiv e^{i\left(\phi_{1 r}-\phi_{2 r}\right)},
$$

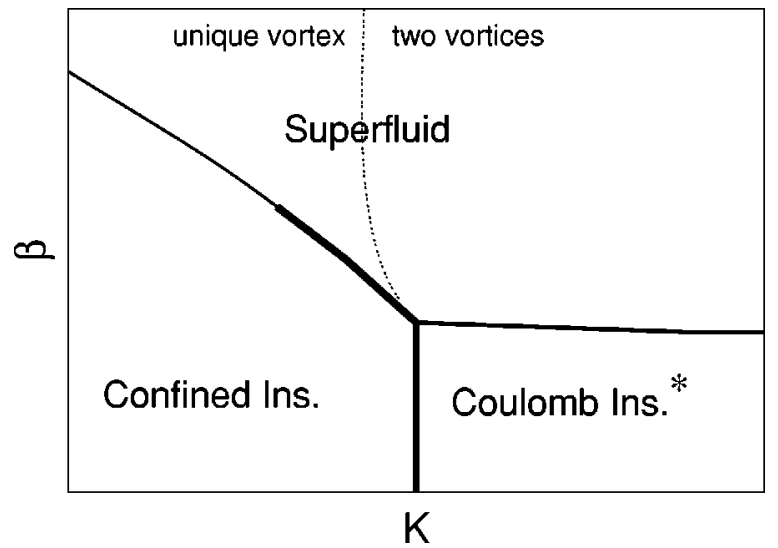

FIG. 5. Schematic phase diagram of the compact $U(1)$ gauge theory with two matter fields, Eq. (5). The dashed line in the superfluid phase represents the conjectured vortex core transition from a unique vortex to two physically distinct vortices (there is no transition in the superfluid ground state across this line).

$$
n_{r}=n_{1 r}=-n_{2 r} .
$$

Applying the corresponding slave-boson mean-field scheme and studying fluctuations about the mean field, one obtains the above gauge theory (the details of the derivation can be found, e.g., in Appendix E of Ref. 15). The constraint, Eq. (10), formally corresponds to the limit $u_{E} \rightarrow \infty$ in $H_{2 c h}$, but we can imagine that some coarse-grained $u_{E}$ is finite, and the description in terms of $b_{1 r}$ and $b_{2 r}$ as emergent degrees of freedom applies in some regime of parameters.

The phase diagram of the two-chargon gauge theory $\mathrm{H}_{2 c h}$ is established by standard arguments ${ }^{16}$ and is shown in Fig. 5 . The different phases are readily identified with the phases of the microscopic boson Hamiltonian, Fig. 4. (i) In the regime $t \ll U, K \gg u_{E}$, the gauge theory is in the deconfined Coulomb phase with gapped chargons; this is of course the Coulomb phase of our microscopic model and has the correct particle content by construction. (ii) In the regime $t \ll U, K$ $\ll u_{E}$, the gauge theory is confining and there are no free gauge charges; only gauge-neutral excitations are free and carry integer boson charge; this phase corresponds to the Mott insulator. (iii) Finally, in the Higgs phase $t \gg U$ both chargon fields condense and the gauge-neutral field $e^{i\left(\phi_{1}-\phi_{2}\right)}$ obtains an expectation value; this corresponds to the superfluid phase of the bosons. It should be emphasized here that while the model gauge theory reproduces the phase diagram of the physical system, it is only in the Coulomb phase that the chargon variables represent low-energy degrees of freedom; in the other phases, the chargons are confined and are not present as individual particles in the spectrum at any energy.

The phenomenological description allows us in particular to discuss the nature of the phase transitions. Thus, we expect the conventional insulator to superfluid transition to be in the $(3+1) \mathrm{D} X Y$ universality (bosons at integer filling) for small $K / u_{E}$, but it can also become first order for larger $K / u_{E}$. The deconfinement transition between the two insulators is similar to that in the pure gauge theory and empirically is believed to be first order. Finally, in the Coulomb to 
superfluid transition the gauge field can be viewed as noncompact since the monopoles remain gapped and do not participate. This transition is expected to be fluctuation-induced first order. ${ }^{17}$

Our treatment so far has been entirely in the boson charge degrees of freedom, and to conclude this discussion we point out some salient properties of the Coulomb phase. First, the Coulomb phase features an emergent gauge charge conservation law that is exact as long as the system remains in this phase. On a formal level, in this case, the projective transformation that has led us to the $U(1)$ gauge theory is convergent as a series in $t / U$, and the result is a generic $U(1)$ gauge theory with the local constraint that is exact by construction. Second, the Coulomb phase and the emergent conservation law are stable against adding arbitrary perturbations to the microscopic Hamiltonian as long as these perturbations are sufficiently small; $5,6,8,10$ this again follows from the local constraint imposed by the large- $U$ term, since the result is by fiat a gauge theory. This amazing fact holds for perturbations that can break all symmetries of the microscopic Hamiltonian and despite the fact that the low-energy theory has a gapless photon. This is a hallmark of the topological structure present in the Coulomb phase.

However, the topological character of the Coulomb phase and its genesis appear mysterious in the charge language. Much further insight is obtained when we consider vortices in the superfluid phase from the point of view of the phenomenological theory.

A precise topological characterization of the Coulomb phase is provided by the existence of the gapped monopole excitation. At energy scales well below the monopole gap, the physics is that of a noncompact $U(1)$ gauge theory. Monopoles remain gapped across the transition to the superfluid phase - as we show below this leads to unusual vortex physics.

Specifically, we argue for the presence of two distinct stable vortices in the superfluid adjacent to the Coulomb phase. We can think of the two vortices as having distinguishable order in the core. Physically, the core can change from one order to the other somewhere along the vortex line, but this domain wall costs an additional energy. The stability of two distinct vortices in some parameter regime is a consequence of some microscopic energetics in the system. When this superfluid state is disordered by proliferating both vortex species, the Coulomb phase obtains, and the domain wall excitations become particles identified with the gapped monopoles.

\section{VORTEX PHENOMENOLOGY AND PHYSICAL PICTURES}

The two-chargon gauge theory is amenable to a precise duality treatment, which gives a dynamical theory of vortices. A formal derivation is given in Appendix A. Here, we proceed gently and consider a semiclassical description of vortices in the superfluid. This discussion provides a physical picture that underlies the more formal treatments of subsequent sections. We argue that, depending on the microscopics, it is possible to have either a unique physical vortex or two distinct vortex species. (A more careful discussion requires the full dynamical theory.) As we vary the parameters in the superfluid phase, there is a transition occurring inside the core of vortex lines from a unique quantum core state to two degenerate but distinct states. The "transition line" cuts the superfluid phase into two regions as indicated in Fig. 5.

It should be emphasized that there is no transition in the ground state of the system. The transition occurs in the properties of an infinite vortex line, which is a very high energy excitation. But this has a bearing on which insulating state is obtained when vortices condense: If we have a unique vortex which then condenses, we obtain the conventional insulator. On the other hand, as we argue below and in subsequent sections, if we have two distinct vortex species and both condense, we obtain the fractionalized Coulomb phase (see Fig. 5). It is also possible to have two vortex species but condense only one of them, and this will give an insulating state with broken discrete symmetry $\left(b_{1} \leftrightarrow b_{2}\right.$ symmetry in the two-chargon model or more physically the sublattice interchange symmetry in the microscopic boson model); in the simple lattice model, Eq. (7), this phase is probably not realized.

We begin with an effective description of the superfluid phase, which is obtained by expanding the cosines in the classical action, Eq. (7); the terms read schematically

$$
\left(\nabla \phi_{1}-a\right)^{2}+\left(\nabla \phi_{2}-a\right)^{2}+(\nabla \times a)^{2} .
$$

Shifting $\boldsymbol{a} \rightarrow \boldsymbol{a}^{\prime}=\boldsymbol{a}-\boldsymbol{\nabla} \phi_{2}$, the field $\boldsymbol{a}^{\prime}$ is massive, and at low energies we are left with one Goldstone mode $\left[\nabla\left(\phi_{1}\right.\right.$ $\left.\left.-\phi_{2}\right)\right]^{2}$, as expected in the superfluid phase.

To study vortices, we apply an external electromagnetic gauge potential that couples to the physical boson number density; this amounts to the replacements

$$
\left(\boldsymbol{\nabla} \phi_{1}-\boldsymbol{a}-\frac{1}{2} \boldsymbol{A}_{\text {ext }}\right)^{2}+\left(\boldsymbol{\nabla} \phi_{2}-\boldsymbol{a}+\frac{1}{2} \boldsymbol{A}_{\text {ext }}\right)^{2}+(\boldsymbol{\nabla} \times \boldsymbol{a})^{2} .
$$

The factors $\pm 1 / 2$ correspond to the $\pm q_{b} / 2$ charges carried by the chargons. An $h c / q_{b}$ vortex is obtained by requiring

$$
\oint \boldsymbol{A}_{\text {ext }} \cdot d \boldsymbol{l}=2 \pi
$$

on going around the vortex. At large distances from the core we have

$$
\boldsymbol{\nabla} \phi_{1}-\boldsymbol{a}-\frac{1}{2} \boldsymbol{A}_{\text {ext }}=0, \quad \boldsymbol{\nabla} \phi_{2}-\boldsymbol{a}+\frac{1}{2} \boldsymbol{A}_{\text {ext }}=0 .
$$

Consider configurations with $\phi_{1,2}$ winding by $2 \pi m_{1,2}$, respectively, $\oint \boldsymbol{\nabla} \phi_{1,2} \cdot d \boldsymbol{l}=2 \pi m_{1,2}$. From the above equations we conclude

$$
\begin{gathered}
\oint \boldsymbol{A}_{\text {ext }} \cdot d \boldsymbol{l}=2 \pi\left(m_{1}-m_{2}\right), \\
\oint \boldsymbol{a} \cdot d \boldsymbol{l}=\pi\left(m_{1}+m_{2}\right) .
\end{gathered}
$$

Thus, there are different ways to realize a physical vortex in terms of the chargon fields. For a unit vortex, the relevant 
realizations are $\left(m_{1}, m_{2}\right)=(1,0)$ and $(0,-1)$ since these have the lowest core energy. Notice that the two realizations carry the gauge field flux $\Phi_{a}=+\pi$ and $-\pi$, respectively. More generally, different realizations of a given vortex are characterized by values of the gauge flux $\Phi_{a}$ that differ by multiples of $2 \pi$. Independent of specific realizations, we note an important distinction between odd-strength vortices, which carry flux $\Phi_{a}=\pi \bmod (2 \pi)$, and even-strength vortices, which carry $\Phi_{a}=0 \bmod (2 \pi)$.

In the above, the gauge field $\boldsymbol{a}$ is treated as noncompact. On a formal level, the multiplicity of the physical vortex appears to be an artifact of ignoring the compactness of the gauge field. Indeed, by moving a monopole across the system, which is a dynamical degree of freedom, we can change the gauge field flux by $2 \pi$. For the preceding discussion of a single vortex, configurations $(1,0)$ and $(0,-1)$ that differ in $\Phi_{a}$ by $2 \pi$ can mix with each other and produce a unique physical vortex (the mixing occurs on the level of local line segments). In this case, the only gauge flux distinction that remains is between even and odd vortices.

However, it is crucial to note that the possibility of the mixing discussed above is a dynamical question. We argue below that it effectively does not occur near the Coulomb phase boundary.

Consider a single physical vortex. As shown above, the vortex carries internal gauge flux of $+\pi$ or $-\pi$ depending on whether it is realized as a vortex in $\phi_{1}$ or $\phi_{2}$. Now consider a "domain wall" inside the core of such a vortex where the internal gauge flux changes from $+\pi$ to $-\pi$. As the internal gauge flux changes by $2 \pi$ at such a domain wall, we may identify it with a monopole configuration of the gauge field. In the Coulomb phase this monopole costs a finite nonzero energy. The monopole gap is expected to stay on approaching the transition to the superfluid. Consequently, close to this phase boundary but in the superfluid side, there will be a finite nonzero energy cost associated with the domain walls inside the vortex core. This immediately implies the existence of two distinct vortex species with the same physical vorticity which are distinguished by the sign of the internal gauge flux in the core.

Upon moving away from the phase boundary between the superfluid and Coulomb phases, the domain wall gap in the vortex core will eventually close. The domain walls will then proliferate inside the core, leading to the destruction of the vortex core order so that there will then be a unique vortex for any given physical vorticity.

What is the physical meaning of the two vortex species? To explore this, first note that the two vortex species may be described as being either a full $2 \pi$ vortex in $b_{1}$ (but not in $b_{2}$ ) or vice versa. Thus the amplitude of $b_{1}$ is suppressed in the core of the $\phi_{1}$ vortex while that of $b_{2}$ is not, and the reverse happens for the $\phi_{2}$ vortex. Thus, the order in the core of the vortex corresponds to spontaneously breaking the symmetry of interchange of $b_{1}$ and $b_{2}$. But what is this symmetry in terms of a more microscopic description in terms of the underlying bosons? The answer clearly depends on the model considered. For the microscopic boson Hubbard model of Sec. II, the two chargon species $b_{1,2}$ are associated with sites that belong to the two distinct sublattices of the cubic lattice on whose links the physical bosons reside. Thus, the chargon interchange symmetry corresponds simply to interchange of the $A$ and $B$ sublattices in the underlying model. We therefore conclude that this sublattice interchange symmetry (present in the microscopic model) is spontaneously broken in the core of a vortex line in the superfluid phase close to the transition to the Coulomb insulator. The two different vortex species simply correspond to the two possible degenerate ordered patterns for the core state.

An almost similar situation also obtains for bosons at half-filling (see Sec. V) on a cubic lattice in a superfluid state close to the transition to a Coulomb insulator. There the two different vortex species again have broken sublattice symmetry in the core which may be simply identified with checkerboard density wave order. This kind of order in the vortex core is very natural for bosons at half-filling - thus a Coulomb insulator is perhaps a more natural possibility at halffilling once the superfluidity is destroyed.

Having understood the vortex structure in the superfluid phase, we can now turn around and address the question of how to view the Coulomb phase in terms of the excitations of the superfluid. To destroy the superfluidity we need to proliferate vortex lines. With two vortex species present, first consider proliferating just one kind of vortex. The resulting insulator will then inherit the order in the core of the proliferated vortex species. In other words, the resulting insulator will break a symmetry (for instance, the sublattice interchange in the boson Hubbard model of Sec. II). On the other hand, we may proliferate both vortex species with equal amplitudes. The resulting insulator will then not inherit the broken symmetry of either vortex core. This is the Coulomb phase. The monopoles are clearly the remnants of the domain walls between the two ordering patterns along the vortex core (see Fig. 2). The photon may then be associated with fluctuations of a "roton" formed by combining together a loop of one vortex species with a loop of an antivortex of the other vortex species. To understand this, we note that in the gauge-theoretic description of the superfluid phase such a roton has no net vorticity but carries internal gauge flux of $2 \pi$. In other words, it represents a tube of magnetic flux. Once the two vortex species proliferate, the fluctuations of the roton may be described in terms of fluctuating magnetic field lines, and this physically describes the photon.

The above physical pictures are elaborated in precise formal terms in the following sections.

\section{DUAL-VORTEX DESCRIPTION}

To make the above discussion of the vortex core structure and its implications for proximate insulating phases more precise, it will clearly be useful to have a dual formulation which focuses on the vortex line degrees of freedom. For the issues at the focus of the present paper-namely, a dual description of the Coulomb phase-it is necessary to have a dual formulation that clearly brings out the presence of the two distinct vortex species with ordered cores. This is most conveniently obtained by dualizing the gauge theoretic description in terms of the two chargon fields. Such a formulation may be obtained by an extension of the duality transformation for the usual lattice $X Y$ model in $(3+1) \mathrm{D}$. This model 
describes bosons at some large integer filling on a threedimensional lattice. Since even this duality is possibly unfamiliar to most readers, we first review it below and show how various familiar Mott insulating states of bosons at integer filling may be described in terms of vortex line condensates. We will then generalize this duality to incorporate the nontrivial core structure of the vortex lines when there are two vortex species and show how the Coulomb phase results from their proliferation.

\section{A. Review of vortex description for $(3+1) D X Y$ model}

Here we review duality for generic 3D quantum bosons at integer filling. The dualities are performed for the Euclidean action, which in this case is $(3+1) \mathrm{D} X Y$ model:

$$
S_{X Y}\left[\phi_{i}\right]=-\beta \sum_{i \mu} \cos \left(\nabla_{\mu} \phi\right) .
$$

To avoid clutter, we take equal spatial and temporal couplings. The formalism is standard, ${ }^{18,19}$ but we want to bring out its physical interpretation as was done in Refs. 11 and 20 for $(2+1) D$. Our treatment below makes explicit the correspondence of the dual variables to vorticities and enables us to obtain a dual perspective on many familiar Mott insulating states. The primary purpose of this review is to provide a background for the subsequent duality treatments of more complicated cases.

We start with the Villain form for the partition function:

$$
Z_{V}=\sum_{\left[p_{i \mu}\right]=-\infty}^{\infty} \int_{-\pi}^{\pi}\left[D \phi_{i}\right] \exp \left[-\frac{\beta}{2} \sum_{i \mu}\left(\nabla_{\mu} \phi-2 \pi p_{i \mu}\right)^{2}\right] .
$$

We work on a $(3+1) \mathrm{D}$ hypercubic lattice; space-time sites are labeled by lowercase Latin letters $(i, \ldots)$, and the lattice directions are labeled by Greek letters $(\mu, \ldots)$. Integervalued fields $p_{i \mu}$ ensure $2 \pi$ periodicity in the angles. For each configuration in the statistical sum, we define vorticities

$$
q_{\mu \nu}=\nabla_{\mu} p_{\nu}-\nabla_{\nu} p_{\mu} .
$$

In $(3+1) \mathrm{D}, q_{\mu \nu}$ describe vortex world sheets. Here and below, the lattice derivatives are indicated only schematically, but the precise meaning is readily recovered in each case.

As usual for $O(2)$ models, we want to separate the spinwave and vortex parts. This is achieved by dividing configurations $\left\{p_{i \mu}\right\}$ into classes with the same vorticity. Two configurations $\left\{p_{i \mu}\right\}$ and $\left\{p_{i \mu}^{\prime}\right\}$ belong to the same class if they can be related via $p_{i \mu}^{\prime}=p_{i \mu}+\nabla_{\mu} N$ with an integer-valued field $N_{i}$. Using the latter, we now extend the $\phi$ integrals over the whole real line and obtain

$$
\begin{aligned}
Z_{V}= & \sum_{\left[q_{i \mu \nu}\right]} \int_{-\infty}^{\infty}\left[D \phi_{i}\right] \int_{-\infty}^{\infty}\left[D j_{i \mu}\right] \exp \left[-\frac{1}{2 \beta} \sum_{i \mu} j_{i \mu}^{2}\right. \\
& \left.+i \sum_{i \mu} j_{i \mu}\left(\nabla_{\mu} \phi-2 \pi p_{i \mu}^{(0)}\right)\right]
\end{aligned}
$$

$$
=\sum_{\left[q_{i \mu \nu}\right]}, \int_{-\infty}^{\infty}\left[D j_{i \mu}\right] \delta\left(\nabla_{\mu} j_{\mu}\right) \exp \left[-\frac{1}{2 \beta} \sum_{i \mu} j_{i \mu}^{2}-i \sum_{i \mu} j_{i \mu} 2 \pi p_{i \mu}^{(0)}\right] .
$$

Here, we introduced a real-valued field $j_{i \mu}$, which can be interpreted as particle current; $\left\{p_{i \mu}^{(0)}\right\}$ is any member of the class with given vorticity $q_{i \mu \nu}$. The prime on the sum over the vorticities $q_{i \mu \nu}$ indicates that these satisfy schematically $d\left(q_{\mu \nu} d x_{\mu} \wedge d x_{\nu}\right)=0$, which reads as an integer-valued constraint

$$
\frac{1}{2} \epsilon_{\rho \sigma \mu \nu} \nabla_{\sigma} q_{\mu \nu}=0
$$

for each direct lattice cube or equivalently each dual lattice link. Here and in what follows, we specifically work in (3 $+1) \mathrm{D}$ and make use of the fully antisymmetric tensor $\epsilon_{\rho \sigma \mu \nu}$ to connect between direct and dual lattice objects; summation over repeated indicies is implied unless specified otherwise. The above constraint means that there are no sources for vortices. We can also specify the vortex world sheets by an integer-valued field $F_{I \rho \sigma}$ residing on the dual plackets (upper case letters $I, \ldots$ specify dual lattice sites):

$$
\begin{gathered}
F_{\rho \sigma}=\frac{1}{2} \epsilon_{\rho \sigma \mu \nu} q_{\mu \nu}, \\
\nabla_{\sigma} F_{\rho \sigma}=0 .
\end{gathered}
$$

The current conservation constraint $\nabla_{\mu} j_{\mu}=0$ can be solved by introducing a rank-2 antisymmetric field $\mathfrak{g}_{I \rho \sigma}$ residing on the dual plackets

$$
j_{\mu}=\frac{1}{2} \epsilon_{\mu \nu \rho \sigma} \nabla_{\nu} \frac{\mathfrak{g}_{\rho \sigma}}{2 \pi},
$$

with factor $2 \pi$ introduced for convenience. We now have

$$
\sum_{i \mu} j_{i \mu} 2 \pi p_{i \mu}^{(0)}=\sum_{I, \rho<\sigma} \mathfrak{g}_{I \rho \sigma} F_{I \rho \sigma},
$$

so vorticity appears explicitly in the statistical sum. At this stage, we can integrate out the field $\mathfrak{g}_{\rho \sigma}$ and obtain a description in terms of the vortex world sheets with specific longranged interactions and consider different phases of the vortex system as determined by this interaction. We expect that modifying the interactions at short distances will not change the physics of the various phases but only positions of the phase boundaries, and we therefore consider generalized models with added local "fugacity" terms

$$
S_{\text {fug }}=\frac{1}{2 \lambda} \sum_{i, \mu<\nu} q_{i \mu \nu}^{2}=\frac{1}{2 \lambda} \sum_{I, \rho<\sigma} F_{I \rho \sigma}^{2} .
$$

In order to describe the vortex system in more familiar terms, we instead retain the $\mathfrak{g}_{\rho \sigma}$ field and proceed as follows. The integer-valued constraint of no vorticity sources written in terms of $F_{I \rho \sigma}$, Eq. (22), is handled by introducing a $U(1)$ variable $c_{I \rho}$ on each dual lattice link in the manner 


$$
\delta\left(\nabla_{\sigma} F_{\rho \sigma}\right) \propto \int_{-\pi}^{\pi} d c_{\rho} \exp \left[i c_{\rho} \nabla_{\sigma} F_{\rho \sigma}\right]
$$

(no sum over $\rho$ ). We can now perform the summation over vorticities, obtaining

$$
\begin{aligned}
Z_{V}[\lambda]= & \int_{-\infty}^{\infty}\left[D \mathfrak{g}_{I \rho \sigma}\right]^{\prime} \int_{-\pi}^{\pi}\left[D c_{I \rho}\right] \exp \left[-\frac{1}{2 \beta} \sum_{i \mu} j_{i \mu}^{2}\right] \\
& \times \exp \left[\lambda \sum_{I, \rho<\sigma} \cos \left(\nabla_{\rho} c_{\sigma}-\nabla_{\sigma} c_{\rho}-\mathfrak{g}_{I \rho \sigma}\right)\right]
\end{aligned}
$$

where the cosine stands for the appropriate Villain form and $j_{\mu}$ is given in Eq. (23). The only approximation in the above duality transformation (besides Villain-izing) is the added vortex fugacity. The resulting dual theory has a compact $U(1)$ gauge field coupled to a noncompact rank-2 field. This is a generalization of the $(2+1) \mathrm{D}$ duality, where the dual theory has a $U(1)$ scalar field coupled to a noncompact gauge field. The vorticity $q_{\mu \nu}$ of the original angle variables is precisely the integer-valued electromagnetic field tensor $F_{\rho \sigma}$ of the compact electrodynamics in the $c_{I \rho}$ variables. In a Hamiltonian formulation, vortex lines are identified with the electric field lines-e.g., $E_{x} \equiv F_{\tau x} \equiv q_{y z}$. Thus, $e^{i c_{R x}}$ is conjugate to $\hat{E}_{R x}$ and can be viewed as a vortex line segment creation operator.

For later convenience, we also exhibit the dual lattice action using "soft-spin" vortex fields

$$
\begin{aligned}
S_{\text {vort }}= & -\lambda \sum_{I, \rho<\sigma}\left[\Psi_{I \rho}^{*} \Psi_{I+\hat{\rho}, \sigma}^{*} \Psi_{I+\hat{\sigma}, \rho} \Psi_{I \sigma} e^{-i \mathfrak{g}_{\rho \sigma}}+\text { c.c. }\right] \\
& +\sum_{I \rho} V\left(\left|\Psi_{I \rho}\right|^{2}\right)+\kappa \sum\left(\epsilon_{\mu \nu \rho \sigma} \nabla_{\nu} \mathfrak{g}_{\rho \sigma}\right)^{2} .
\end{aligned}
$$

Here $\Psi_{I \rho}^{*} \sim e^{i c_{I \rho}}$ creates a vortex line segment on the dual lattice link; $V\left(|\Psi|^{2}\right)=s|\Psi|^{2}+u_{4}|\Psi|^{4}+u_{6}|\Psi|^{6}+\cdots$ controls amplitude fluctuations of the field $\Psi$. From the preceding derivation, the rank-2 field $\mathfrak{g}_{I \rho \sigma}$ describes boson density fluctuations via Eq. (23). The first term in the action represents vortex line "hopping" by extending across a placket. The boson density acts as a source for the rank-2 gauge potential seen by the vortices (this gauge potential produces the familiar Berry phase when a vortex line moves in the superfluid).

The behavior of the compact $U(1)$ gauge theory without the rank-2 field is well understood, and we can use this as a starting point to develop intuition about the vortex system. This line of thinking can be interpreted as considering screened vortices first. Indeed, for a charged boson system we would conclude that the field $\mathfrak{g}_{\rho \sigma}$ is massive and can be ignored. To obtain a faithful description of neutral bosonse.g., to correctly reproduce the low-energy modes-we need to include the "gauging" by the rank-2 field. This can be accomplished by a semiclassical analysis just as in the Ginzburg-Landau theory.

We now summarize the dual description of the familiar phases of 3D quantum bosons at integer filling.

The superfluid phase of the bosons is identified with confinement in $e^{i c_{\rho}}$. Vortex excitations in the superfluid are the gapped electric field lines. The rank- 2 field $\mathfrak{g}_{\rho \sigma}$ is essentially free, and its single propagating mode (which can be found, e.g., by doing quantum mechanics for this field) is precisely the phonon mode of the superfluid.

The Mott insulator phase is obtained by proliferating vortices, so $e^{i c_{\rho}}$ is "condensed" or, more appropriately, "deconfined." The rank-2 field $\mathfrak{g}_{\rho \sigma}$ is gapped out and in the process "eats" the photon modes (an analog of the Anderson-Higgs phenomenon), so there are no gapless excitations, as expected. Gapped charged excitations of the Mott insulator are represented by monopoles of the dual gauge field, and the discreteness of charge is encoded in the corresponding quantization condition for the rank-2 field, which is a generalization of the flux quantization in the Ginzburg-Landau theory.

Indeed, let us consider a monopole worldline. Fix a time slice (of our Euclidean path integral) and evaluate the boson number

$$
n_{\mathrm{tot}}=\int j_{\tau} d^{3} r=\frac{1}{2 \pi} \int d^{3} r\left(\nabla_{x} \mathfrak{g}_{y z}+\nabla_{y} \mathfrak{g}_{z x}+\nabla_{z} \mathfrak{g}_{x y}\right) .
$$

In the far field away from the monopole we have, e.g.,

$$
\mathfrak{g}_{x y}=\nabla_{x} c_{y}-\nabla_{y} c_{x} \equiv B_{z},
$$

so the right-hand side coincides with the outgoing magnetic flux divided by $2 \pi$, which is equal to the enclosed monopole number. Thus, monopole world lines in the dual description are precisely world lines of integer-quantized charges in the direct description.

$Z_{2}$ fractionalized insulator: We can readily generalize the above description of the Mott insulator to discuss more exotic insulator phases. The $Z_{2}$ fractionalized phase is obtained as a condensate of doubled vortices while single vortices are not condensed. The dual action generically allows doublevortex hopping terms like

$$
-\lambda_{2} \sum_{I, \rho<\sigma} \cos \left[2\left(\nabla_{\rho} c_{\sigma}-\nabla_{\sigma} c_{\rho}-\mathfrak{g}_{I \rho \sigma}\right)\right] .
$$

When $\lambda$ is small while $\lambda_{2}$ is large, we expect $e^{i 2 c_{\rho}}$ to be deconfined but not $e^{i c_{\rho}}$. Topological excitations in this phase are monopoles of the field $e^{i 2 c_{\rho}}$, and these are seen to represent charged excitations carrying fraction $1 / 2$ of the boson charge. Odd-strength vortex line excitations correspond to vison line excitations of the $Z_{2}$ phase.

\section{B. Dual formulation with two vortex species}

We now show how to incorporate the physics of two distinct vortex species with ordered cores in the dual formulation. As mentioned above, this is most conveniently done by dualizing the gauge-theoretic description in terms of two chargon fields. The duality transformation is a straightforward but tedious extension of the methods of the previous subsection. We therefore relegate the technical details to Appendix A and focus here on the result and the physics contained in it. As expected on the basis of the semiclassical discussion in Sec. III, the analysis in Appendix A yields a dual theory with two vortex fields $\Psi^{(1)}$ and $\Psi^{(2)}$ corresponding to the two possible ordering patterns in the core. Further, these vortices are allowed to turn from one flavor into the 
other, and the corresponding domain wall appears as an additional field $Y$ in the dual theory.

A generic "soft-spin" action for the two vortex fields can be expressed in the form

$$
\begin{aligned}
S_{2 \mathrm{vort}}= & -\lambda \sum_{b=1,2}\left[\Psi_{I \rho}^{(b) *} \Psi_{I+\hat{\rho}, \sigma}^{(b) *} \Psi_{I+\hat{\sigma}, \rho}^{(b)} \Psi_{I \sigma}^{(b)} e^{-i \mathfrak{g}_{\rho \sigma}}+\text { c.c. }\right] \\
& +\sum\left[V\left(\left|\Psi_{I \rho}^{(1)}\right|^{2}+\left|\Psi_{I \rho}^{(2)}\right|^{2}\right)+W\left(\left|\Psi_{I \rho}^{(1)}\right|^{2}\left|\Psi_{I \rho}^{(2)}\right|^{2}\right)\right] \\
& +\kappa \sum\left(\epsilon_{\mu \nu \rho \sigma} \nabla_{\nu} \mathfrak{g}_{\rho \sigma}\right)^{2}-\lambda_{m} \sum\left(\Upsilon_{I}^{*} \Upsilon_{I+\hat{\rho}} \Psi_{I \rho}^{(1) *} \Psi_{I \rho}^{(2)}\right. \\
& + \text { c.c. })+\sum U\left(\left|\Upsilon_{I}\right|^{2}\right) .
\end{aligned}
$$

In the two-chargon theory, both $\Psi_{I \rho}^{(1) *}$ and $\Psi_{I \rho}^{(2)^{*}}$ create a physical vortex line segment, but it is realized as a vortex in $b_{1}$ in the first case and an antivortex in $b_{2}$ in the second case; see Eq. (13). The vortex interaction terms $V$ and $W$ need not be specified at this stage but are important when determining the vortex condensate. $\lambda_{m}$ represents quantum tunneling between the two core states and is written here as the domain wall hopping, while $U$ represents the domain wall energy cost.

Two broad possibilities need to be considered in the superfluid. The first possibility is that domain walls are energetically cheap and proliferate inside vortex cores. In this case the two vortex fields lock to each other. In the integer filling case considered here, this produces a unique physical vortex, and the proper low-energy description is then in terms of a single vortex field (the situation at half-integer filling is more complicated; see the next section).

The second possibility is that domain walls are energetically costly and remain gapped inside vortex cores. In this case the field $Y$ has only short-ranged correlations and may be integrated out. The two vortex fields are proper lowenergy degrees of freedom. We can now ask what happens when these vortices condense-e.g., when $\Psi=0$ becomes an unstable state of the potential $V$. Depending on the interaction $W$, the condensate either has just one of the two vortex species or has both vortex species proliferated simultaneously. The former case occurs when $W>0$, and we write schematically $\left\langle\Psi^{(1)}\right\rangle \neq 0,\left\langle\Psi^{(2)}\right\rangle=0$. The resulting Mott insulator then inherits the order in the core of the $\Psi^{(1)}$ vortex. There are no gapless modes: as discussed in the previous subsection, the rank-2 field Higgses out the gapless modes of the single-species world sheet condensate. In the microscopic boson Hubbard model on the lattice of corner-sharing octahedra, this Mott insulator breaks $A \leftrightarrow B$ sublattice interchange symmetry.

On the other hand, for the interaction $W$ of opposite sign, it is favorable for both vortex species to proliferate simultaneously, schematically $\left\langle\Psi^{(1)}\right\rangle=\left\langle\Psi^{(2)}\right\rangle \neq 0$. In this case we obtain a fractionalized Coulomb insulator with a gapless photon, a gapped monopole, and two species of gapped chargons. The gapless photon obtains since the rank-2 field $\mathfrak{g}_{\rho \sigma}$ can Higgs out only the $\Psi^{(1)^{*}} \Psi^{(2)^{*}}$ 'part' of the twospecies world sheet condensate. The $\Psi^{(1)^{*}} \Psi^{(2)}$ part does not couple to $\mathfrak{g}_{\rho \sigma}$; it represents string objects with short-range interactions only-indeed, it is precisely the roton formed by combining a vortex of one kind with an antivortex of the other kind. To show that its fluctuations lead to a gapless photon mode, we focus on just the fluctuations of the "phases" $\mathcal{C}^{(1)}, C^{(2)}$ of the two vortex species:

$$
\Psi^{(1)^{*}} \sim e^{i \mathcal{C}^{(1)}}, \quad \Psi^{(2) *} \sim e^{i \mathcal{C}^{(2)}} .
$$

(Note that compared with Appendix A, we use $\mathcal{C}^{(1)}=c^{(1)}$, $\mathcal{C}^{(2)}=-c^{(2)}$, while the monopole field $\theta^{(m)}$ is the "phase" of the domain wall particle $Y_{I}^{*} \sim e^{i \theta_{I}^{(m)}}$.) Expanding to quadratic order in small gradients of $\mathcal{C}^{(1)}, \mathcal{C}^{(2)}$, the resulting 'elastic' action has the following schematic structure:

$$
\begin{aligned}
S= & \left(\boldsymbol{\nabla} \times \mathcal{C}^{(1)}-\mathfrak{g}\right)^{2}+\left(\boldsymbol{\nabla} \times \mathcal{C}^{(2)}-\mathfrak{g}\right)^{2} \\
& +\left[\boldsymbol{\nabla} \times\left(\mathcal{C}^{(1)}-\mathcal{C}^{(2)}\right)\right]^{2}+\cdots
\end{aligned}
$$

Here $\mathfrak{g}$ represents the rank-2 gauge field. The ellipses refer to the kinetic energy term for the $\mathfrak{g}$ and various anharmonic corrections to the above quadratic action. It is now easy to see that the combination $\mathcal{C}^{(1)}+\mathcal{C}^{(2)}$ is rendered massive while the combination $\mathcal{C}^{(1)}-\mathcal{C}^{(2)}$ remains massless. The latter precisely represents a linear dispersing gapless photon with two transverse polarizations in three spatial dimensions. Thus as promised the photon is indeed associated with fluctuations of the roton formed from a vortex of one kind and antivortex of the other kind. The gapped $Y$ field simply corresponds to the monopole excitation of the Coulomb phase. Finally, the "monopoles" of the proliferated dual fields $\Psi^{(1)}$ and $\Psi^{(2)}$ are the two chargons. This completes our formal description. We also point that Appendix D develops a similar description of the Ising $P^{*}$ phase in two spatial dimensions that incorporates the physics of two distinct Ising domain walls.

We conclude by emphasizing that the vortex proliferation transition is likely first order, in which case the lattice action for vortices does not have a formal continuum limit, but this does not affect the presented physical picture of the phases.

\section{BOSONS AT HALF-FILLING}

We now consider bosons at half-filling in some detail. We have in mind some generic Hamiltonian

$$
H_{\text {generic }}=u_{b} \sum_{r}\left(n_{r}-\frac{1}{2}\right)^{2}-w_{b} \sum_{r r^{\prime}} b_{r}^{\dagger} b_{r^{\prime}}+\cdots \text {. }
$$

The physical picture presented in the Introduction and elaborated in the previous section carries over readily and is perhaps more appealing in this case. In particular, the ordering pattern in the vortex core when there are two species simply corresponds to checkerboard density order of the bosons. This is a very natural "insulating" core for the vortices to develop when the bosons are at half-filling. Formally, this core structure may readily be seen to arise in the same semiclassical treatment as in Sec. III. We first break up the boson field into two "chargon" fields as before. This leads to a description in terms of a compact $U(1)$ gauge theory coupled to two chargon fields. A physical vortex in the superfluid 
order then corresponds to having a full vortex in $b_{1}$ (but none in $b_{2}$ ) or a full antivortex in $b_{2}$ (and no vorticity in $b_{1}$ ). Thus, there are still two types of vortices which convert into each other at the locations of monopoles of the gauge theory. Following the closely related discussion in Ref. 15 in two dimensions, it is readily seen that the two vortices correspond to the two possible patterns of checkerboard density-wave ordering in the core. (The precise reason is simply that the two vortices differ in the sign of the internal gauge flux. For bosons at half-filling, this gauge flux couples linearly to the difference of the boson densities on the two sublattices; hence there is checkerboard density-wave ordering in the core).

As for bosons at integer filling, the insight into the vortex structure can be given a precise form by employing a duality transformation. The dual action is derived in Appendix C. The Coulomb phase is again obtained when both vortex species condense with equal amplitude. The domain walls in the ordered vortex core survive as gapped monopoles, and the photon emerges exactly as before (as the fluctuations of proliferated rotons made by combining a vortex and an antivortex of two different species). The gapped chargons are again "monopoles" of the two vortex fields that appear in the dual theory. Thus the main structure of the description of the Coulomb phase is unaffected from that appropriate at integer filling.

The primary differences arise when we consider the nature of the possible confined phases. These may be obtained from the Coulomb phase by condensing the gapped monopoles. Such a condensation leads to confinement of the gapped chargons and the disappearance of the photon from the spectrum. However, as can be anticipated from experience in two dimensions, the monopoles now transform nontrivially under lattice symmetries. This leads to broken lattice symmetry in the confined phases. In particular, various bond or box density wave phases are possible. These will be explored directly in the next section.

Here we specify more precisely the modifications to the two-vortex action, Eq. (32), due to half-filling on a cubic lattice and sketch some implications for the vortex core structure. As discussed in Appendix C, there is an additional static vector potential $X_{I \rho}^{0}$ that couples to the domain wall hopping term-the $\lambda_{m}$ term in Eq. (32) - see Eq. (C4). This vector potential encodes the monopole Berry phases due to half-integer boson density. It is specified by the corresponding fluxes $\mathfrak{h}_{\rho \sigma}^{0}=\nabla_{\rho} X_{\sigma}^{0}-\nabla_{\sigma} X_{\rho}^{0}$ (modulo $2 \pi$ ) through the faces of the dual cubes. The fluxes are nonzero on the spatial plackets only and equal to $\pi / 3$ when oriented from the $A$ to $B$ sublattice of the direct cubic lattice as shown in Fig. 6. The derivation of this result is given in the next section, and further details can be found in Appendix C.

As discussed above, the two fields $\Psi^{(1)}$ and $\Psi^{(2)}$ correspond to the two checkerboard charge orders inside a vortex core. We can now ask about the effect on the core dynamics due to the static vector potential $X_{\rho}^{0}$ seen by the domain walls. The specific details of the domain wall motion such as change in the dispersion are not immediately relevant for the low-energy physics if the domain wall remains gapped.
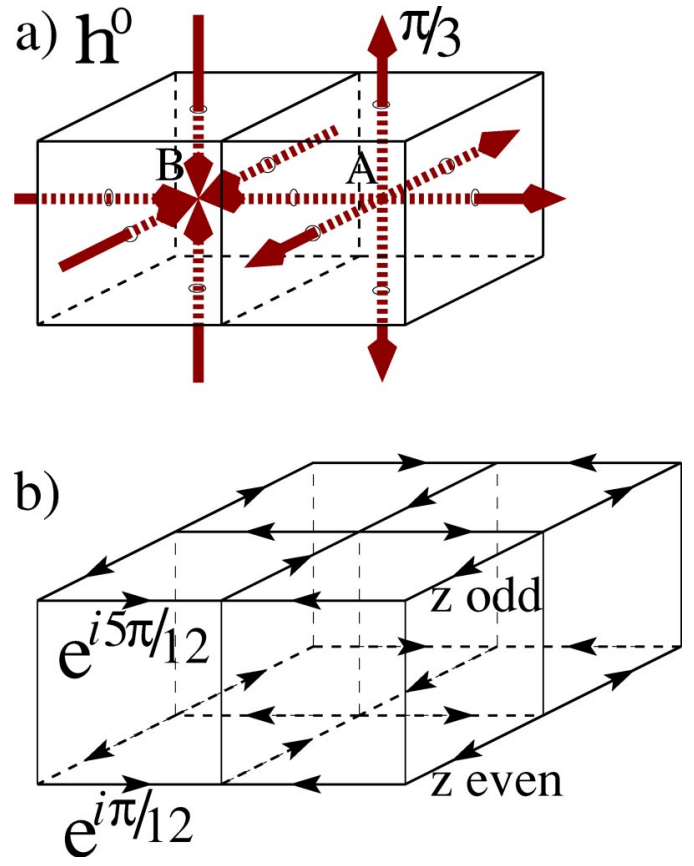

FIG. 6. Description of the monopole Berry phases for bosons at half-filling on a simple cubic lattice in terms of a static gauge potential seen by the monopoles hopping on the dual lattice. (a) $\mathfrak{h}_{\rho \sigma}^{0}$ gives a flux of $\pm \pi / 3$ through each spatial placket of the dual lattice with the flux oriented from the $A$ to $B$ sublattice of the direct lattice. (b) Our gauge choice for $e^{i X_{\rho}^{0}}$, which realizes the fluxes $e^{i h_{\rho \sigma}^{0}}$ (Eq. (46)).

Indeed this is why the modifications induced by half-filling do not affect much the description of the Coulomb phase itself. But they become more important when the monopole gap closes at a phase transition from the Coulomb to a confined phase or when the domain wall gap closes inside vortex core.

Deep in the superfluid phase when the vortex line is stiff, we expect a single minimum in the domain wall dispersion, and when the walls proliferate inside the core we obtain a unique vortex. However, for a vortex line that fluctuates sufficiently and explores the three-dimensional space so that the effects of $X_{\rho}^{0}$ are felt, the bottom of the domain wall band can split. This is the situation in the Coulomb phase, where domain walls become monopole particles, and one finds two low-energy propagating monopole modes (see the next section for details).

Consider now what happens when we have two domain wall band minima inside the vortex core in the superfluid phase and the domain wall gap goes to zero. One possibility motivated by our analysis in the next section is that domain wall proliferation leads to a VBS order inside the core. In this case, while the fields $\Psi^{(1)}$ and $\Psi^{(2)}$ are locked, there can be several degenerate ways for this to happen corresponding to the number of favorable VBS states inside the core (we need to be even more careful here since, e.g., for the columnar order the favorable states likely depend on the vortex line orientation). This would mean that our choice of the basic fields $\Psi^{(1)}$ and $\Psi^{(2)}$ is not a very good one and the analysis 
needs to be reconsidered, perhaps introducing different vortex fields. When a single such vortex condenses, the corresponding VBS state results, but it may be also possible to condense several such vortices simultaneously possibly leading to new exotic states. Even more exotic possibility is for the domain walls to become critical inside the core, which might occur due to the one dimensionality of the setting. ${ }^{21}$ In the present work, we have only touched upon these possibilities and have not pursued any systematic studies.

We now comment on the possibility of a direct analysis of a more "microscopic" vortex theory for bosons at half-filling and the connection with the above two-vortex description. Indeed such a direct analysis is very useful in two spatial dimensions. ${ }^{14,15}$ The bare vortex action at half-filling is described in Appendix B. As in $(2+1) \mathrm{D}$, the half-filling manifests itself by "frustrating" the vortex propagation with additional static Berry phases. In $(3+1) \mathrm{D}$, this is encoded by placing half of a magnetic monopole inside each dual cube in the compact gauge field part that describes the vortex motion. As mentioned in Appendix B, at present we do not know how to analytically treat such frustrated gauge theories and how to connect directly with the two-vortex description like it was done in Ref. 14 for $(2+1) \mathrm{D}$. Our earlier discussion and the analysis in Appendix $\mathrm{C}$ provide an alternative route in two dimensions ${ }^{15}$ that extends reasonably to three dimensions, and we expect the resulting action with two vortex species to describe the vortex physics at low energies in some regime of parameters. (A limited but direct attack is possible on the bare vortex action as well. ${ }^{21}$ )

\section{VALENCE BOND SOLIDS OF BOSONS AT HALF- FILLING ON A 3D CUBIC LATTICE}

In this section, we show how valence bond solid phases emerge due to monopole condensation out of the Coulomb phase at half-filling. The crucial ingredient is the monopole dynamics produced by the static vector potential $X_{\rho}^{0}$. To that end it is convenient to specialize to the hard-core limit in which case we may view the bosons as representing spin- $1 / 2$ moments residing on the cubic lattice. In this limit, the static vector potential may be viewed as arising from Berry phases present in a path integral description of a spin-1/2 moment. Because of the importance of this result, which is an extension to three dimensions of Haldane's calculation in two dimensions ${ }^{22}$ we give a direct derivation in the context of spin-1/2 systems. It is in this context that the valence bond solids were analyzed in the work of Read and Sachdev, ${ }^{23}$ providing us with familiar grounds.

\section{A. Monopole action and Berry phases}

Our starting point is the "modified" Sachdev-Jalabert ${ }^{15,24,25}$ lattice model for spin-1/2 system on a cubic lattice in the vicinity of the collinear Néel state. This is defined in terms of a bosonic field $z_{i \alpha}$ which carries spin $1 / 2$ and resides on the sites (denoted $i, j, \ldots)$ of a $(3+1) \mathrm{D}$ space-time lattice ( $\alpha=\uparrow, \downarrow$ is a spin index). We will refer to the quanta of this field as spinons. They are the analog (for the spin system) of the chargon fields introduced earlier for boson models. The spinons are minimally coupled to a compact $U(1)$ gauge field $a_{i \mu}$. The action for the SachdevJalabert model reads

$$
\begin{gathered}
Z_{S J}=\sum_{\left[u_{i \mu \nu}\right]} \int_{-\pi}^{\pi}\left[D a_{i \mu}\right] \int\left[D \mathbf{z}_{i}\right]\left[\delta\left(\left|\mathbf{z}_{i}\right|^{2}-1\right)\right] \\
\quad \times \exp \left(-S_{z}-S_{a}-S_{B}\right), \\
S_{z}=-\beta \sum_{i \mu}\left[\mathbf{z}_{i}^{\dagger} e^{\left.i a_{i \mu} \mathbf{Z}_{i+\hat{\mu}}+\text { c.c. }\right]+\cdots}\right. \\
S_{a}=\frac{K}{2} \sum_{i, \mu<\nu}\left(\nabla_{\mu} a_{\nu}-\nabla_{\nu} a_{\mu}-2 \pi u_{i \mu \nu}\right)^{2} \\
S_{B}=i \sum_{i} \eta_{i} a_{i \tau} .
\end{gathered}
$$

The compactness of the gauge field is encapsulated by summing over integer-valued fields $u_{i \mu \nu}$. The last term represents a Berry phase that encapsulates the spin-1/2 nature of the moment at each site. The $\eta_{i}$ appearing in this term is +1 on the $A$ sublattice and -1 on the $B$ sublattice of the spatial cubic lattice. The connection to microscopic spin models and motivation may be found in the original references 23-25. With appropriate deformations of $S_{z}$, this model can also describe bosonic systems at half-integer filling (see Refs. 15 and 25).

Here we are interested in paramagnetic states with gapped spinons (interpreted as chargons in bosonic systems). In such cases we may integrate out the spinon fields completely. This effectively amounts to dropping the $S_{z}$ term from the action. (As we are interested in the general structure of the paramagnetic phases, we will not worry much about effects such as generating other short ranged interactions for the gauge fields.) The remaining action is that of a compact $U(1)$ gauge theory in $(3+1) \mathrm{D}$ on a cubic lattice but in the presence of the Berry phase term. The latter has a simple physical interpretation in a Hamiltonian language. It simply corresponds to the presence of a static background gauge charge of strength \pm 1 on the sites of the spatial cubic lattice. The sign alternates from one sublattice to the other. Indeed precisely the same kind of gauge theory arises in studies of two-dimensional spin-1/2 quantum paramagnets.

In the $(2+1) \mathrm{D}$ case, it is well known that the alternating static background charge leads to broken translational symmetry in confined paramagnetic phases. Our goal is to extend that analysis to three dimensions. To that end we perform a duality transformation on $S_{a}+S_{B}$. In the absence of background charge, this is just the familiar electric-magnetic duality. The compact $U(1)$ gauge theory then becomes a theory of pointlike monopoles that are coupled to the dual fluctuating noncompact $U(1)$ gauge field. (The noncompactness of the dual gauge field is because we have thrown out the spinons-which are the gauge "electric" charges-in the original theory. Indeed retaining them is equivalent to retaining monopoles in the dual gauge field.) The presence of the background charge means that there is dual magnetic flux emanating out of the center of each cube on the dual lattice 
on which the monopoles reside. This flux alternates in sign from one cube to the next and frustrates the monopole hopping. We therefore expect the duality transformation on $S_{a}$ $+S_{B}$ to yield a theory of monopoles with frustrated hopping that is coupled to the dual noncompact gauge field. This is explicitly shown in what follows.

We proceed by writing

$$
\begin{aligned}
e^{-S_{a}}= & \int_{-\infty}^{\infty}\left[D f_{i \mu \nu}\right] \exp \left(-\frac{1}{2 K} \sum_{i, \mu<\nu} f_{i \mu \nu}^{2}\right) \\
& \times \exp \left[i \sum_{i, \mu<\nu} f_{i \mu \nu}\left(\nabla_{\mu} a_{\nu}-\nabla_{\nu} a_{\mu}-2 \pi u_{i \mu \nu}\right)\right] .
\end{aligned}
$$

$f_{\mu \nu}$ can be interpreted as the electromagnetic field tensor. We classify configurations $\left\{u_{i \mu \nu}\right\}$ by their monopole four-currents

$$
J_{\rho}^{(m)}=\frac{1}{2} \epsilon_{\rho \sigma \mu \nu} \nabla_{\sigma} u_{\mu \nu} .
$$

Two configurations $\left\{u_{\mu \nu}\right\}$ and $\left\{u_{\mu \nu}^{\prime}\right\}$ belong to the same class if they can be related, $u_{\mu \nu}^{\prime}=u_{\mu \nu}+\nabla_{\mu} V_{\nu}-\nabla_{\nu} V_{\mu}$, with an integer-valued field $V_{\mu}$. This allows the separation of the Gaussian and topological defect parts of the statistical sum, since the $a_{\mu}$ integration now extends over the full real line.

To treat the Berry phase term, we find a static $f_{\mu \nu}^{0}$ satisfying

$$
\begin{gathered}
\eta_{i} \delta_{\mu \tau}=\nabla_{\nu} f_{\mu \nu}^{0}, \\
f_{\mu \nu}^{0}=-\frac{n_{0} K}{\beta}\left(\delta_{\mu \tau} \nabla_{\nu} \eta_{i}-\delta_{\nu \tau} \nabla_{\mu} \eta_{i}\right) .
\end{gathered}
$$

(As will be clear below, the proportionality constant $n_{0} K / \beta$ is in fact fixed by the above conditions to be $1 / 12$; the specific writing is chosen for convenience when reusing the present analysis in Appendix C.) The first condition is possible since $v_{i \mu} \equiv \eta_{i} \delta_{\mu \tau}$ has zero divergence. It allows us to write the Berry phase term as

$$
S_{B}=i \sum_{\mu<\nu} f_{\mu \nu}^{0}\left(\nabla_{\mu} a_{\nu}-\nabla_{\nu} a_{\mu}\right) .
$$

We can now bring out the topological defect part by changing variables to $\tilde{f}_{\mu \nu}=f_{\mu \nu}-f_{\mu \nu}^{0}$. In terms of the variables $\tilde{f}_{\mu \nu}$ the action has the same form as in the absence of the original Berry phases except for additional contributions:

$$
S_{n_{0}}+\widetilde{S}_{B} \equiv \frac{1}{K} \sum_{\mu<\nu} \tilde{f}_{\mu \nu} f_{\mu \nu}^{0}+i \sum_{\mu<\nu} f_{\mu \nu}^{0} 2 \pi u_{\mu \nu} .
$$

Due to condition (38) the "phase" term $\widetilde{S}_{B}$ depends on the monopole configuration only. The second condition (39) allows us to write the term $S_{n_{0}}$ as

$$
S_{n_{0}}=\sum_{i} \frac{n_{0}}{\beta} \eta_{i} j_{i \tau}
$$

Here $j_{\mu} \equiv \nabla_{\nu} \tilde{f}_{\mu \nu}$ can be viewed as the gauge charge current. This follows by integrating over the $a_{\mu}$ field; Appendixes A and $\mathrm{C}$ exhibit this explicitly for $U(1)$ matter fields, while in the absence of any matter we get $\nabla_{\nu} \widetilde{f}_{\mu \nu}=0$. Thus, $S_{n_{0}}$ can be viewed as a static staggered chemical potential seen by the $\mathbf{z}$ fields, and as long as it is much smaller than the spinon gap, it can be ignored for the low-energy properties.

We now exhibit $f_{\mu \nu}^{0}$ that solves the two conditions, Eqs. (38) and (39). Only the electric field parts $E_{x}^{0} \equiv f_{\tau x}^{0}, E_{y}^{0}$, and $E_{z}^{0}$ are nonzero and satisfy

$$
\begin{gathered}
\boldsymbol{\nabla} \cdot \boldsymbol{E}^{0}=\eta_{r}, \\
\boldsymbol{E}^{0}=-\frac{K n_{0}}{\beta} \boldsymbol{\nabla} \eta_{r} .
\end{gathered}
$$

This fixes $\boldsymbol{E}^{0}$ uniquely to have value $1 / 6$ on each bond from the $A$ to $B$ sublattice (the value $n_{0} / \beta$ is also fixed at this stage).

The phase term $\widetilde{S}_{B}$ can be expressed in terms of the monopole currents $J_{\rho}^{(m)}$ by first transforming from $f_{\mu \nu}^{0}$ to $\mathfrak{h}_{\rho \sigma}^{0}$ :

$$
f_{\mu \nu}^{0}=\frac{1}{2} \epsilon_{\mu \nu \rho \sigma} \frac{\mathfrak{h}_{\rho \sigma}^{0}}{2 \pi}
$$

The latter has spatial components $\mathfrak{h}_{x y}^{0}, \mathfrak{h}_{y z}^{0}, \mathfrak{h}_{z x}^{0}$ only, which can be interpreted as fluxes through the faces of the dual lattice cubes. The total outwards flux is $+2 \pi$ for a dual cube surrounding an $A$-sublattice site of the direct lattice and $-2 \pi$ surrounding a $B$-sublattice site, and in each case the flux is divided equally among all six faces. This is shown in Fig. 6 (a). Since the total flux out of each cube is $\pm 2 \pi$, we can write

$$
\mathfrak{h}_{\rho \sigma}^{0}=\nabla_{\rho} X_{\sigma}^{0}-\nabla_{\sigma} X_{\rho}^{0} \bmod 2 \pi
$$

$X_{\rho}^{0}$ is a static field on the spatial links of the dual lattice, and one choice is shown in Fig. 6(b). We finally obtain

$$
\tilde{S}_{B}=i \sum J_{\rho}^{(m)} X_{\rho}^{0}
$$

and interpret this as a monopole Berry phase. This is appropriate since as we have explained the remaining contributions to the low-energy action have the same form as in the absence of the original Berry phase $S_{B}$.

We can offer the following geometric representation of the monopole Berry phase. Consider a monopole space-time world line and trace out the spatial path traveled by the monopole. The Berry phase is given by the flux of $\mathfrak{h}^{0}$ (see Fig. 6) through any surface pulled onto this closed path. This is $(3+1) \mathrm{D}$ generalization of Haldane's $(2+1) \mathrm{D}$ result ${ }^{22}$ for the monopole Berry phase. In $(2+1) \mathrm{D}$, the Haldane's result leads to destructive interference among monopole world paths unless monopoles are quadrupled. In $(3+1) \mathrm{D}$, the interference pattern encoded in $\mathfrak{h}_{\rho \sigma}^{0}$ is more subtle, and requires us to study the monopole hopping in the static vector potential $X_{\rho}^{0}$; this is detailed below.

We now complete the duality mapping for the model $S_{a}$ $+S_{B}$ without any matter field in order to exhibit the relevant 
structure of the monopole action; the relationship of this to the original Sachdev-Jalabert model will be commented upon later.

Using the standard result for the monopole action in compact QED we obtain

$$
\begin{aligned}
Z_{\mathrm{cqed}+S_{B}}= & \sum_{\left[J_{\rho}^{(m)}\right]}, \int_{-\infty}^{\infty}\left[D L_{\rho}\right]^{\prime} \\
& \times \exp \left(-\frac{1}{8 K \pi^{2}} \sum_{\rho<\sigma}\left(\nabla_{\rho} L_{\sigma}-\nabla_{\sigma} L_{\rho}\right)^{2}\right) \\
& \times \exp \left(-i \sum J_{\rho}^{(m)}\left(L_{\rho}+X_{\rho}^{0}\right)\right)
\end{aligned}
$$

Here, $L_{\rho}$ is a noncompact dual gauge field such that $\tilde{f}_{\mu \nu}$ $=\epsilon_{\mu \nu \rho \sigma} \nabla_{\rho} L_{\sigma} /(2 \pi)$. The prime on the sum indicates that the monopole currents satisfy continuity $\nabla_{\rho} J_{\rho}^{(m)}=0$. Integrating over $L_{\rho}$ we would obtain an action in terms of the monopole world lines only. Instead of doing this, we transform the monopole action into a more familiar form by considering a generalized model with added monopole fugacity term

$$
S_{\text {fug. }}=\frac{1}{2 \lambda_{m}} \sum\left(J_{\rho}^{(m)}\right)^{2} .
$$

The constraint $\nabla_{\rho} J_{\rho}^{(m)}(I)=0$ on each dual site $I$ can be solved by introducing a $U(1)$ field $\theta_{I}^{(m)}$, and upon summing over $J_{\rho}^{(m)}$ the final result reads

$$
\begin{aligned}
Z_{\text {cqed }+S_{B}}\left[\lambda_{m}\right]= & \int_{-\infty}^{\infty}\left[D L_{I \rho}\right]^{\prime} \int_{-\pi}^{\pi}\left[D \theta_{I}^{(m)}\right] e^{-S\left[L_{\rho}, \theta^{(m)}\right]}, \\
S\left[L_{\rho}, \theta^{(m)}\right]= & \frac{1}{8 K \pi^{2}} \sum_{\rho<\sigma}\left(\nabla_{\rho} L_{\sigma}-\nabla_{\sigma} L_{\rho}\right)^{2} \\
& -\lambda_{m} \sum \cos \left(\nabla_{\rho} \theta^{(m)}-X_{\rho}^{0}-L_{\rho}\right) .
\end{aligned}
$$

$e^{i \theta^{(m)}}$ can be interpreted as a monopole creation operator; the field $\theta^{(m)}$ is coupled to the noncompact (dual) gauge field $L_{\rho}$, and there is also static "frustration" $X_{\rho}^{0}$ coming from the Berry phases for monopoles in our spin- $1 / 2$ system.

The relationship of the above action to the original Sachdev-Jalabert model is as follows. The action was derived in the absence of the spinon field $\mathbf{z}$ (e.g., taking the spinon gap to infinity). Now, $\mathbf{z}$ carries electric charge of the original gauge field $a_{\mu}$, and as usual in the electromagnetic duality, it acts as a magnetic charge for the dual gauge field $L_{\rho}$. Thus, to have a complete correspondence with the original spin model, we need to allow monopoles in the dual gauge field $L_{\rho}$, and these monopoles need to be spin-1/2 particles. Ignoring the spinon field makes the dual gauge field noncompact as in the above action.

\section{B. Monopole condensation patterns}

We now study possible phases of the above monopole action, assuming throughout that the spinons are gapped.
Clearly, for small $\lambda_{m}$ the monopole field is gapped. In this case, the dual gauge field is free, and we obtain a $U(1)$ spin liquid phase of the original spin- $1 / 2$ problem. This phase has gapped spinons, gapped monopoles, and a gapless photon and respects all lattice symmetries.

To analyze possible monopole condensates, we closely follow Ref. 14. We first ignore fluctuations of the dual-gauge field $L_{\rho}$ and study a frustrated $X Y$ model defined by the static vector potential $X_{\rho}^{0}$. What we are essentially doing here is averaging over fast fluctuations imposed by $X_{\rho}^{0}$. Once the structure of the resulting slow fluctuation description becomes clear, we will restore the field $L_{\rho}$.

Continuous-time soft-spin action for the frustrated $X Y$ model is

$$
\int d \tau\left[\sum_{R}\left|\partial_{\tau} \Phi_{R}\right|^{2}-\sum_{\left\langle R R^{\prime}\right\rangle}\left(t_{R R^{\prime}} \Phi_{R}^{*} \Phi_{R^{\prime}}+\text { c.c. }\right)+\sum_{R} V\left(\left|\Phi_{R}\right|^{2}\right)\right],
$$

with some potential $V\left(\left|\Phi_{R}\right|^{2}\right)=r_{0}\left|\Phi_{R}\right|^{2}+u_{0}\left|\Phi_{R}\right|^{4}+\cdots$. We work on the discrete spatial lattice labeled by $R$ (dual to the original spin system lattice), since the lattice is crucial at this stage.

The frustration is encoded in the monopole hopping am-

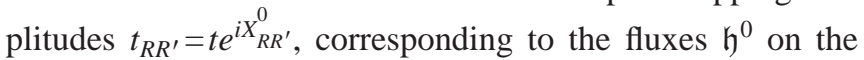
plackets as shown in Fig. 6(a). Our gauge choice is shown in Fig. 6(b), and details are follows:

$$
\begin{gathered}
t_{R, R+\hat{x}}=\sqrt{\frac{3}{8}}\left(1+i e^{i \pi(x+y)}\right)+\sqrt{\frac{1}{8}}\left(1-i e^{i \pi(x+y)}\right) e^{i \pi z}, \\
t_{R, R+\hat{y}}=\sqrt{\frac{3}{8}}\left(1-i e^{i \pi(x+y)}\right)+\sqrt{\frac{1}{8}}\left(1+i e^{i \pi(x+y)}\right) e^{i \pi z}, \\
t_{R, R+\hat{z}}=1 .
\end{gathered}
$$

Diagonalizing the kinetic energy, we find two low-energy modes with normalized real-space wave functions

$$
\begin{gathered}
\Psi_{1}(R)=\frac{1+(\sqrt{3}-\sqrt{2}) e^{i \pi z}}{\sqrt{2(3-\sqrt{6})}}, \\
\Psi_{2}(R)=\frac{1-(\sqrt{3}-\sqrt{2}) e^{i \pi z}}{\sqrt{2(3-\sqrt{6})}} \times \frac{e^{i \pi x}-i e^{i \pi y}}{\sqrt{2}} .
\end{gathered}
$$

Thus, we find two monopole excitations carrying different lattice momenta.

At the kinetic energy level, any linear combination

$$
\Phi(R)=\phi_{1} \Psi_{1}(R)+\phi_{2} \Psi_{2}(R)
$$

is at the bottom of the monopole band, and there is a continuum of states for the monopoles to condense to. Nonlinear terms will lift the degeneracy. This can be analyzed near the monopole condensation transition by treating $\phi_{1}$ and $\phi_{2}$ as slowly varying fields and deriving Ginzburg-Landau theory of these.

By examining the action of the lattice symmetries, the resulting Ginzburg-Landau functional is required to be in- 
variant under the following transformations (in our specific gauge):

$$
\begin{gathered}
T_{x}: \phi_{1} \rightarrow \phi_{1}^{*}, \quad \phi_{2} \rightarrow-\phi_{2}^{*} ; \\
T_{y}: \phi_{1} \rightarrow \phi_{1}^{*}, \quad \phi_{2} \rightarrow \phi_{2}^{*} ; \\
T_{z}: \phi_{1} \rightarrow \phi_{2}^{*}, \quad \phi_{2} \rightarrow \phi_{1}^{*} ; \\
R_{90^{\circ}, R x y}: \phi_{1} \rightarrow e^{-i \pi / 4} \phi_{1}^{*}, \quad \phi_{2} \rightarrow e^{i \pi / 4} \phi_{2}^{*} ; \\
R_{90^{\circ}, R x z}: \phi_{1} \rightarrow \frac{\phi_{1}^{*}+\phi_{2}^{*}}{\sqrt{2}}, \quad \phi_{2} \rightarrow \frac{\phi_{1}^{*}-\phi_{2}^{*}}{\sqrt{2}} .
\end{gathered}
$$

The $90^{\circ}$ rotations are about the lattice points on which monopoles reside. Note that the realization of the above lattice symmetries also involves conjugation $\phi$ to $\phi^{*}$ (followed by appropriate gauge transformations).

The simplest invariants are $\left|\phi_{1}\right|^{2}+\left|\phi_{2}\right|^{2}$ and

$$
4\left(\left|\phi_{1}\right|^{6}\left|\phi_{2}\right|^{2}+\left|\phi_{1}\right|^{2}\left|\phi_{2}\right|^{6}\right)-6\left|\phi_{1}\right|^{4}\left|\phi_{2}\right|^{4}-\left(\phi_{1}^{*} \phi_{2}\right)^{4}-\left(\phi_{1} \phi_{2}^{*}\right)^{4} \text {. }
$$

The latter can be given a more clear form

$$
I_{8}\left(\phi_{1}, \phi_{2}\right)=N_{x}^{2} N_{y}^{2}+N_{y}^{2} N_{z}^{2}+N_{z}^{2} N_{x}^{2},
$$

with

$$
N_{\alpha}\left(\phi_{1}, \phi_{2}\right) \equiv \phi^{\dagger} \hat{\sigma}^{\alpha} \phi
$$

We write down the continuum action for the twocomponent complex field $\phi(R, \tau)$, which respects the above symmetries,

$$
S_{\text {slow }}=\int d \tau d^{3} R\left[\left|\left(\nabla_{\rho}-i L_{\rho}\right) \phi\right|^{2}+U\left(|\phi|^{2}\right)+v_{8} I_{8}(\phi)\right]+S_{L},
$$

with some potential $U\left(|\phi|^{2}\right)=r|\phi|^{2}+u_{4}|\phi|^{4}+\cdots$. We have also restored the dual gauge field $L_{\rho}$ and included some generic kinetic energy $S_{L}$ for $L_{\rho}$.

We focus on confining paramagnets that obtain starting from the Coulomb phase and condensing single monopoles so that $\langle\phi\rangle \neq 0$; this happens when $r<0$ in the potential. [It is also possible to have paired condensates such that $\left\langle\phi^{\dagger} \vec{\sigma} \phi\right\rangle$ $\neq 0$ but $\langle\phi\rangle=0$ in some parameter regime-such states correspond to $U(1)$ spin liquids with broken translational symmetry; these are not considered in any detail here.] Ground states are selected by minimizing $v_{8} I_{8}(\phi)$, and the sign of $v_{8}$ determines the character of the resulting phase.

Each state can be characterized by the expectation values $N_{x}, N_{y}, N_{z}$ defined above. For example, the spatial monopole density in a given state is given by

$$
|\Phi(R)|^{2}=|\phi|^{2}+\frac{1}{\sqrt{3}}\left[(-1)^{x} N_{x}+(-1)^{y} N_{y}+(-1)^{z} N_{z}\right] ;
$$

the monopole kinetic energy is
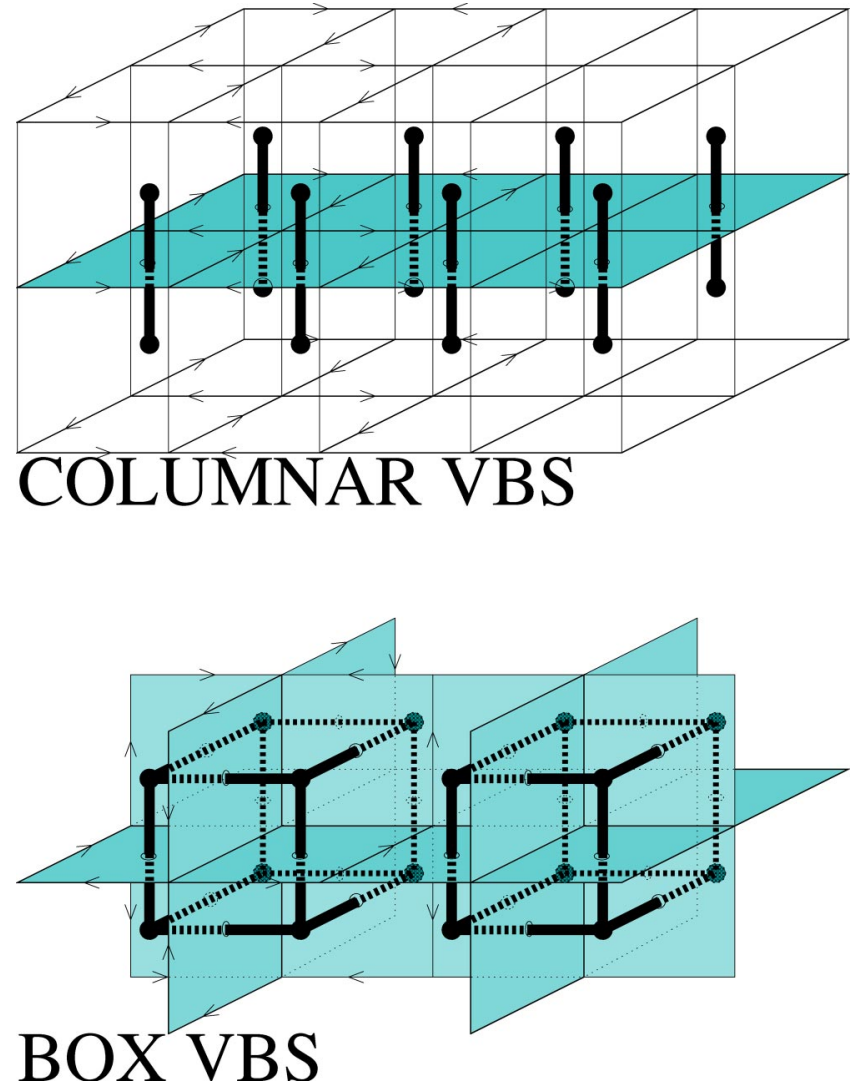

FIG. 7. (a) Schematic picture of the columnar valence bond solid (VBS) state obtained when $v_{8}>0$. We have an increased monopole density (and also energy density) on the shaded $z$-even planes, which is interpreted as having valence bonds of the original spin model preferentially crossing these planes. Arrows along the placket edges indicate monopole currents. (b) 3D box VBS state is obtained when $v_{8}<0$. For clarity, only the planes with increased monopole density are shown $(x, y, z$ all even), and the dimers resonate around the cubes centered where these planes meet.

$$
E_{x}^{(m)}=\sqrt{\frac{8}{3}}|\phi|^{2}+\sqrt{2}\left[(-1)^{y} N_{y}+(-1)^{z} N_{z}\right]
$$

for $E_{x}^{(m)} \equiv t_{R, R+\hat{x}} \Phi_{R}^{*} \Phi_{R+\hat{x}}+$ c.c., etc., while the monopole current is

$$
J_{x}^{(m)}=\sqrt{\frac{2}{3}}\left[(-1)^{x+z} N_{y}-(-1)^{x+y} N_{z}\right]
$$

for $J_{x}^{(m)} \equiv i\left(t_{R, R+\hat{x}} \Phi_{R}^{*} \Phi_{R+\hat{x}}-\right.$ c.c. $)$.

We also quote the transformation properties of $\vec{N}$ $=\left(N_{x}, N_{y}, N_{z}\right)$ under the lattice symmetries

$$
\begin{gathered}
T_{x}:\left(N_{x}, N_{y}, N_{z}\right) \rightarrow\left(-N_{x}, N_{y}, N_{z}\right) ; \\
T_{y}:\left(N_{x}, N_{y}, N_{z}\right) \rightarrow\left(N_{x},-N_{y}, N_{z}\right) ; \\
T_{z}:\left(N_{x}, N_{y}, N_{z}\right) \rightarrow\left(N_{x}, N_{y},-N_{z}\right) ; \\
R_{90^{\circ}, R x y}:\left(N_{x}, N_{y}, N_{z}\right) \rightarrow\left(N_{y}, N_{x}, N_{z}\right) ;
\end{gathered}
$$




$$
R_{90^{\circ}, R x z}:\left(N_{x}, N_{y}, N_{z}\right) \rightarrow\left(N_{z}, N_{y}, N_{x}\right) .
$$

Given the above information, we can characterize broken lattice symmetries when $\vec{N}$ obtains an expectation value.

$$
\text { 1. } v_{8}>0
$$

In this case, there are six ground states

$$
\left(N_{x}, N_{y}, N_{z}\right)=( \pm 1,0,0), \quad(0, \pm 1,0), \quad(0,0, \pm 1) .
$$

In a given state, the monopole density is the same in every other plane perpendicular to a fixed lattice axis. For example, the state $\left(N_{x}, N_{y}, N_{z}\right)=(0,0,1)$ has an increased density on the $z$-even planes and decreased density on the $z$-odd planes and is illustrated in Fig. 7(a). In the original spin model, these dual planes are crossed by direct lattice bonds, and on a crude level there is an increased bond energy crossing the $z$-even planes. Thus, this state corresponds to a columnar valence bond solid with dimers oriented in the $\hat{z}$ direction and in the particular registry. This identification is also supported by an analysis of the dual-gauge-field fluxes induced by the monopole currents and the connection between these fluxes and the bond order parameter. ${ }^{23}$ The above six states correspond to six possible columnar states on the cubic lattice. We expect that this is the phase realized when monopoles condense in the specific lattice model, Eq. (50), based on the analysis of the lattice $X Y$ model and the large- $\lambda_{m}$ limit of the action, Eq. (50).

$$
\text { 2. } v_{8}<0
$$

In this case, there are eight ground states

$$
\left(N_{x}, N_{y}, N_{z}\right)=\frac{1}{\sqrt{3}}( \pm 1, \pm 1, \pm 1)
$$

(each of the three signs can be chosen independently). These states have monopole density oscillating in all three directions and correspond to three-dimensional "box" valence bond solids analogous to box states in two dimensions. For example, the state $\left(N_{x}, N_{y}, N_{z}\right)=(1 / \sqrt{3})(1,1,1)$ has maximal monopole density for $x, y$, and $z$ all even and is illustrated in Fig. 7(b). In the original spin model, this state has dimers resonating around direct lattice cubes surrounding these dual lattice points. The above eight states correspond to eight possible ways to register such 3D box states.

\section{Discussion}

The above considerations lead us to the following picture. In the spin-1/2 system we expect VBS order parameter to be a three-vector

$$
\vec{\Psi}_{V B S}=\left(\begin{array}{c}
(-1)^{x} \vec{S}_{r} \cdot \vec{S}_{r+\hat{x}} \\
(-1)^{y} \vec{S}_{r} \cdot \vec{S}_{r+\hat{y}} \\
(-1)^{z} \vec{S}_{r} \cdot \vec{S}_{r+\hat{z}}
\end{array}\right)
$$

We can write down a Ginzburg-Landau functional for $\vec{\Psi}$ :
$S_{V B S}=\int d \tau d^{3} r\left[\left(\nabla_{\mu} \vec{\Psi}\right)^{2}+r \vec{\Psi}^{2}+u\left(\vec{\Psi}^{2}\right)^{2}+v\left(\Psi_{x}^{4}+\Psi_{y}^{4}+\Psi_{z}^{4}\right)\right]$

where besides $O(3)$-invariant terms we have also included quartic interaction with cubic anisotropy $v$, since our system resides on the cubic lattice. Thus, the VBS phases in three dimensions are described by an effective $O(3)$ model with cubic anisotropy. Depending on the sign of the anisotropy, the three-vector $\vec{\Psi}$ can either point along one of the axes [e.g., $\vec{\Psi} \propto(0,0,1)]$, which leads to columnar order with six degenerate states, or along a diagonal [e.g., $\vec{\Psi} \propto(1,1,1) / \sqrt{3}]$, which leads to box order with eight degenerate states.

The connection with the preceding analysis is provided by the identification

$$
\vec{\Psi}_{V B S} \sim \vec{N}=\phi^{\dagger} \vec{\sigma} \phi
$$

Indeed, $\vec{\Psi}$ has the same transformation properties under lattice symmetries as $\vec{N}$ [see Eq. (61)—we recall that there the rotations are about points $R$ of the lattice on which monopoles reside, which is dual to the original spin model lattice]. Thus, the three-vector $\vec{\Psi}$ is written with the help of the twocomponent complex field $\phi$ precisely as in the $C P^{1}$ representation of the $O(3)$ nonlinear $\sigma$ model. As we know, a proper description of the $O(3)$ model in the $C P^{1}$ language contains besides the spinor field $\phi$ also a compact $U(1)$ gauge field, and the monopoles of this gauge field are conventionally identified with the hedgehog configurations of the $O(3)$ vector. This $C P^{1}$ gauge field is precisely our gauge field $L$ in the $\phi$-field action, Eq. (57), and as discussed earlier, for a faithful description of the original spin-1/2 system we need to allow spin-1/2 carrying magnetic monopoles in the gauge field $L$, which correspond to the gapped spinons.

The identification is complete when we realize, extending the discussion in a recent preprint by Levin and Senthil, ${ }^{26}$ that the VBS order parameter field $\vec{\Psi}_{V B S}$ indeed has hedgehogs which are precisely the spinons. This is dictated by the microscopic lattice origin of $\vec{\Psi}_{V B S}$, which needs to be brought back into consideration [this microscopic detail is absent in the continuum action $S_{V B S}$, Eq. (65)]. Consider, for example, the columnar phase and construct a hedgehog in the field $\vec{N}$ by putting together the corresponding domains of columnar dimer order. More precisely, we are constructing a "hedgehog" of the corresponding six-state discrete model. When we join these domains, there is an unpaired site left which we identify with a spinon residing on one sublattice; and if we construct an antihedgehog, we find a spinon residing on the other sublattice. Of course, we pay huge domain wall energies consistent with the fact that the spinons are confined in the VBS phase.

\section{Connection with the vortex description}

We conclude by describing how to obtain the above picture directly from the two-vortex action, Eqs. (32) and (C4), at half-filling. We use hard-spin fields and notation as in 
Appendixes A and C. Consider phases with gapped chargons obtained by proliferating both vortex fields $c^{(1)}$ and $c^{(2)}$. Ignoring the chargons completely, we can expand the cosines containing $\boldsymbol{\nabla} \times c$ and, after simple analysis, arrive at the monopole action, Eq. (50), with $L_{\rho}=c_{\rho}^{(1)}+c_{\rho}^{(2)}$. The real utility of the two-vortex action is that it allows us to incorporate the chargons on equal footing in the dual description as "monopoles" in the vortex fields $c^{(1)}$ and $c^{(2)}$. In particular, it formalizes for the easy-plane case the purported picture that we are to include monopoles in the gauge field $L_{\rho}$ to obtain the full physical description; no such formalism is known for the Heisenberg case.

\section{CONCLUSIONS}

In this paper, we described a physical mechanism for the appearance of the fractionalized Coulomb phase in bosonic models. This was accomplished by addressing the question of how to view this phase as a vortex condensate emanating out of a proximate superfluid phase. We were led to suggest one route whereby there are two degenerate but distinct physical vortices in the superfluid phase, and the superfluid is destroyed by proliferating both species of vortices with equal amplitude. The two vortex species correspond to vortices whose cores (which are one-dimensional systems) are ordered and break a discrete symmetry. A simple example is provided by bosons at half-filling where the vortex core develops insulating checkerboard charge density order. The two vortex species correspond to the two checkerboard states in the core. Domain walls in the core order survive as gapped excitations when the two vortex species proliferate equally. We argued that these domain walls may be identified with the monopole excitations of the Coulomb phase. Roton loops formed by combining a vortex loop with one core order with an antivortex loop with the other core order were shown to correspond to loops of the emergent magnetic flux. The fluctuations of these loops correspond to the gapless photon excitation of the Coulomb phase.

In general, the microscopics that produces degenerate but distinct physical vortices is model specific. But quite broadly, we can view these vortices as having ordered cores, where the order is that of some nearby insulating phase with a broken discrete symmetry. For generic bosons at half-filling, one simple candidate is the checkerboard charge order, while for the specific corner-sharing octahedra model discussed in Sec. II the candidate has checkerboard "octahedron" order.

The primary analytical tool used in the paper to give substance to this description is a duality mapping to vortex variables which is an extension of the familiar boson-vortex duality to $(3+1) \mathrm{D}$. We extensively developed such a description for the quantum systems of interest and used it to discuss the physics. To discuss the Coulomb phases, we showed how the presence of two vortex species can be readily incorporated into the dual theory. The excitation spectrum of the Coulomb phase was then derived in this dual formulation which provides detailed confirmation of the physical pictures. This theory also allows us to identify and describe all basic nearby insulating states in a unified setting.

For bosons at half-filling (closely related to spin-1/2 quantum antiferromagnets), apart from the fractionalized phases, various confining phases are possible which break lattice translation symmetry. The latter were analyzed by extension to $(3+1) \mathrm{D}$ of the methods of Haldane ${ }^{22}$ and Read and $\mathrm{Sachdev}^{23}$ (originally developed for lower dimension). This allowed us to discuss possible valence bond orders in this system and their relation to the fractionalized Coulomb insulating state.

In the present study of bosonic superfluids and proximate insulators at half-filling, we concentrated on vortices with charge-ordered cores. It is conceivable instead to have valence bond order inside the core in some regime of parameters in the superfluid phase, and it would be interesting to develop a broader picture of the core dynamics and its consequences. The above considerations provide further explicit examples of nontrivial effects arising from the vortex core physics for disordering transitions of $X Y$ ordered systems, echoing similar phenomena in two-dimensional systems. ${ }^{15,26}$ In particular, we showed that there can be transformations occurring inside the core and that proliferating ordered cores can produce unusual phases. The importance of the core physics may have broader implications for experimental studies of strongly correlated systems.

\section{ACKNOWLEDGMENTS}

Stimulating discussions with Leon Balents, Matthew Fisher, Mike Hermele, and Ashvin Vishwanath are gratefully acknowledged. This research is supported by NSF Grant No. PHY-9907949 (O.I.M.) and NSF Grant No. DMR-0308945 (T.S.). T.S. also acknowledges funding from the NEC Corporation, the Alfred P. Sloan Foundation, and an award from The Research Corporation.

\section{APPENDIX A: DUALITY TRANSFORMATION FOR TWO- CHARGON GAUGE THEORY, Eq. (7)}

Here we perform a duality analysis of the two-chargon gauge theory, Eq. (7). Our final result is the two-vortex action, Eq. (32), at integer filling. We first identify topological defects in the model. The Villain-ized partition function for the model is written as

$$
\begin{aligned}
Z_{V}= & \int_{-\pi}^{\pi}\left[D \phi_{1 i} D \phi_{2 i} D a_{i \mu}\right] \sum_{\left[p_{1 i \mu}, p_{2 i \mu}, u_{i \mu \nu}\right]=-\infty}^{\infty} \int_{-\infty}^{\infty}\left[D j_{1 i \mu} D j_{2 i \mu} D f_{i \mu \nu}\right] \exp \left(-\frac{1}{2 \beta} \sum\left(j_{1}^{2}+j_{2}^{2}\right)-\frac{1}{2 K_{i, \mu<\nu}} \sum_{i \mu \nu} f_{i}^{2}\right) \\
& \times \exp \left\{i \sum\left[\boldsymbol{j}_{1} \cdot\left(\boldsymbol{\nabla} \phi_{1}-\boldsymbol{a}-2 \pi \boldsymbol{p}_{1}\right)+\boldsymbol{j}_{2} \cdot\left(\boldsymbol{\nabla} \phi_{2}-\boldsymbol{a}-2 \pi \boldsymbol{p}_{2}\right)\right]+i \sum_{i, \mu<\nu} f_{i \mu \nu}\left(\nabla_{\mu} a_{\nu}-\nabla_{\nu} a_{\mu}-2 \pi u_{i \mu \nu}\right)\right\} .
\end{aligned}
$$


The real fields $\boldsymbol{j}_{1}, \boldsymbol{j}_{2}, f_{\mu \nu}$ can be interpreted as the matter currents and the electromagnetic field tensor.

We divide configurations $\left\{\boldsymbol{p}_{1}, \boldsymbol{p}_{2}, u_{\mu \nu}\right\}$ into classes equivalent under integer-valued transformations,

$$
\begin{gathered}
\boldsymbol{p}_{b}^{\prime}=\boldsymbol{p}_{b}+\nabla N_{b}-\boldsymbol{V}, \quad b=1,2, \\
u_{\mu \nu}^{\prime}=u_{\mu \nu}+\nabla_{\mu} V_{\nu}-\nabla_{\nu} V_{\mu},
\end{gathered}
$$

with integer fields $N_{1}, N_{2}, \boldsymbol{V}$. These classes are specified by two vorticities

$$
q_{\mu \nu}^{(b)}=\nabla_{\mu} p_{b \nu}-\nabla_{\nu} p_{b \mu}+u_{\mu \nu}
$$

and monopole currents

$$
J_{\rho}^{(m)}=\frac{1}{2} \epsilon_{\rho \sigma \mu \nu} \nabla_{\sigma} u_{\mu \nu}
$$

The latter satisfy the continuity

$$
\nabla_{\rho} J_{\rho}^{(m)}=0
$$

while the vorticities satisfy $d q^{(b)}=d u$, which can be written as an integer-valued constraint

$$
\frac{1}{2} \epsilon_{\rho \sigma \mu \nu} \nabla_{\sigma} q_{\mu \nu}^{(b)}=J_{\rho}^{(m)}, \quad b=1,2 .
$$

The meaning of the last equation is that monopoles act as sources and sinks for both vorticities simultaneously.

For each allowed configuration of vorticities and monopole currents, we can now perform the summation over all $\left\{\boldsymbol{p}_{1}, \boldsymbol{p}_{2}, u_{\mu \nu}\right\}$ in the corresponding class using Eqs. (A2). This effectively extends the integration variables $\phi_{1}, \phi_{2}, a_{\mu}$ over the whole real line, and the integrals give the $\delta$-function conditions

$$
\begin{gathered}
{\left[\delta\left(\boldsymbol{\nabla} \cdot \boldsymbol{j}_{1}\right)\right]\left[\delta\left(\boldsymbol{\nabla} \cdot \boldsymbol{j}_{2}\right)\right]\left[\delta\left(\nabla_{\nu} f_{\mu \nu}-j_{1 \mu}-j_{2 \mu}\right)\right]} \\
\propto\left[\delta\left(\boldsymbol{\nabla} \cdot \boldsymbol{j}^{-}\right)\right]\left[\delta\left(j_{\mu}^{+}-\nabla_{\nu} f_{\mu \nu}\right)\right] .
\end{gathered}
$$

In the last line, we changed variables to $\boldsymbol{j}^{ \pm}=\boldsymbol{j}_{1} \pm \boldsymbol{j}_{2}$. Note that not all conditions in the first line are independent, and the precise meaning of the $\delta$ functions is given by the second line. Thus, we can completely eliminate $\boldsymbol{j}^{+}$.

The topological defects enter through

$$
\begin{aligned}
i \pi & \sum\left(\boldsymbol{j}^{+}\left(\boldsymbol{p}_{1}+\boldsymbol{p}_{2}\right)+\boldsymbol{j}^{-}\left(\boldsymbol{p}_{1}-\boldsymbol{p}_{2}\right)\right)+i 2 \pi \sum_{\mu<\nu} f_{\mu \nu} u_{\mu \nu} \\
& =i \pi \sum_{\mu<\nu} f_{\mu \nu}\left(q_{\mu \nu}^{(1)}+q_{\mu \nu}^{(2)}\right)+i \pi \sum \boldsymbol{j}^{-}\left(\boldsymbol{p}_{1}-\boldsymbol{p}_{2}\right) .
\end{aligned}
$$

We now solve the constraint $\boldsymbol{\nabla} \cdot \boldsymbol{j}^{-}=0$ with a rank-2 field $\mathfrak{g}_{I \rho \sigma}^{-}$ defined on the dual plackets

$$
j_{\mu}^{-}=\frac{1}{2} \epsilon_{\mu \nu \rho \sigma} \nabla_{\nu} \frac{\mathfrak{g}_{\rho \sigma}^{-}}{\pi} .
$$

It is also convenient to pass from the variables $f_{\mu \nu}$ to their dual $\mathfrak{g}_{\rho \sigma}^{+}$via

$$
f_{\mu \nu}=\frac{1}{2} \epsilon_{\mu \nu \rho \sigma} \frac{\mathfrak{g}_{\rho \sigma}^{+}}{\pi}
$$

$\boldsymbol{j}^{+}$is obtained from $\mathfrak{g}^{+}$by an expression similar to Eq. (A7). Finally, it is convenient to specify vorticities $q_{\mu \nu}^{(b)}$ by the corresponding integer-valued fields $F_{\rho \sigma}^{(b)}, b=1,2$,

$$
F_{\rho \sigma}^{(b)}=\frac{1}{2} \epsilon_{\rho \sigma \mu \nu} q_{\mu \nu}^{(b)}, \quad \nabla_{\sigma} F_{\rho \sigma}^{(b)}=J_{\rho}^{(m)},
$$

just as we did for the $(3+1) \mathrm{D} X Y$ model in Eq. (22).

Putting everything together, the partition sum reads

$$
\begin{aligned}
Z= & \sum_{\left[F_{I \rho \sigma}^{(1)}, F_{I \rho \sigma}^{(2)}, J_{I \rho}^{(m)}\right]}^{\prime} \int_{-\infty}^{\infty}\left[D \mathfrak{g}_{I \rho \sigma}^{+}\right]\left[D \mathfrak{g}_{I \rho \sigma}^{-}\right]^{\prime} \\
& \times \exp \left(-\frac{1}{4 \beta} \sum\left(j_{+}^{2}+j_{-}^{2}\right)-\frac{1}{2 K \pi^{2}} \sum_{\rho<\sigma}\left(\mathfrak{g}_{\rho \sigma}^{+}\right)^{2}\right) \\
& \times \exp \left(-i \sum_{\rho<\sigma}\left[\mathfrak{g}_{\rho \sigma}^{+}\left(F^{(1)}+F^{(2)}\right)_{\rho \sigma}+g_{\rho \sigma}^{-}\left(F^{(1)}-F^{(2)}\right)_{\rho \sigma}\right]\right) .
\end{aligned}
$$

We can now in principle integrate out the fields $\mathfrak{g}_{\rho \sigma}^{+}$and $\mathfrak{g}_{\rho \sigma}^{-}$, and obtain a theory in terms of vortex world sheets with sources and sinks on the monopole world lines. The vortex interactions are such that combinations $F^{(1)}+F^{(2)}$ enter with short-ranged interactions similar to screened vortices, while $F^{(1)}-F^{(2)}$ enter with long-ranged interactions of unscreened vortices. This is expected since the chargon field combination $\phi_{1}+\phi_{2}$ is gauged while $\phi_{1}-\phi_{2}$ is gauge neutral. We expect the physics to remain unchanged upon adding local vortex and monopole fugacity terms

$$
S_{\text {fug. }}=\frac{1}{2 \lambda} \sum_{I, \rho<\sigma}\left[\left(F_{I \rho \sigma}^{(1)}\right)^{2}+\left(F_{I \rho \sigma}^{(2)}\right)^{2}\right]+\frac{1}{2 \lambda_{m}} \sum_{I \rho}\left(J_{I \rho}^{(m)}\right)^{2} .
$$

Just as in the case with the $X Y$ model in Sec. IV A, to get a better intuition about the theory at hand, we instead introduce two compact $U(1)$ gauge fields $c_{I \rho}^{(1)}, c_{I \rho}^{(2)}$ and a $U(1)$ scalar field $\theta_{I}^{(m)}$ that implement the constraints Eqs. (A9) and (A5) and are the appropriate conjugate variables.

The final expression reads 


$$
\begin{aligned}
Z_{V}\left[\lambda, \lambda_{m}\right]= & \int_{-\pi}^{\pi}\left[D c_{I \rho}^{(1)} D c_{I \rho}^{(2)} D \theta_{I}^{(m)}\right] \int_{-\infty}^{\infty}\left[D \mathfrak{g}_{I \rho \sigma}^{+}\right]\left[D \mathfrak{g}_{I \rho \sigma}^{-}\right]^{\prime} \exp \left(-\frac{1}{4 \beta} \sum\left(j_{+}^{2}+j_{-}^{2}\right)-\frac{1}{2 K \pi^{2}} \sum_{\rho<\sigma}\left(\mathfrak{g}_{\rho \sigma}^{+}\right)^{2}\right) \\
& \times \exp \left[\lambda_{m} \sum_{I \rho} \cos \left(\nabla_{\rho} \theta^{(m)}-c_{I \rho}^{(1)}-c_{I \rho}^{(2)}\right)\right. \\
& \left.+\lambda \sum_{I, \rho<\sigma} \cos \left(\nabla_{\rho} c_{\sigma}^{(1)}-\nabla_{\sigma} c_{\rho}^{(1)}-\mathfrak{g}_{I \rho \sigma}^{+}-\mathfrak{g}_{I \rho \sigma}^{-}\right)+\lambda \sum_{I, \rho<\sigma} \cos \left(\nabla_{\rho} c_{\sigma}^{(2)}-\nabla_{\sigma} c_{\rho}^{(2)}-\mathfrak{g}_{I \rho \sigma}^{+}+\mathfrak{g}_{I \rho \sigma}^{-}\right)\right] .
\end{aligned}
$$

As usual, cosines stand for the appropriate Villain forms. At this stage, the field $\mathfrak{g}_{\rho \sigma}^{+}$is massive and can be integrated out. The variables of the resulting dual theory are (i) two compact $U(1)$ gauge fields $e^{i c^{(1)}}$ and $e^{i c^{(2)}}$, which can be viewed as vortex line segment creation operators, (ii) a monopole matter field $e^{i \theta^{(m)}}$ that carries both dual gauge charges, and (iii) a noncompact rank-2 field $\mathfrak{g}^{-}$that further "gauges" the vortex fields $c^{(1)}$ and $c^{(2)}$ and describes the physical boson density fluctuations. Equation (32) in Sec. IV B displays a soft-spin version of this dual theory by writing

$$
\Psi_{I \rho}^{(1)^{*}} \sim e^{i c_{I \rho}^{(1)}}, \quad \Psi_{I \rho}^{(2)^{*}} \sim e^{-i c_{I \rho}^{(2)}}, \quad \Upsilon_{I}^{*} \sim e^{i \theta_{I}^{(m)}}
$$

(we also dropped the superscript on the rank-2 gauge field $\mathfrak{g}^{-}$ that survives in the low-energy theory).

The analysis of the possible phases from this dual perspective can be done as in the $X Y$ case in Sec. IV A by first considering a theory without the $\mathfrak{g}^{-}$field (i.e., "screened vortex" theory which would obtain if the two chargons were also coupled to a fluctuating external electromagnetic field). The field $\mathfrak{g}^{-}$is then included semiclassically and provides correct count of the low-energy modes.

Thus, the screened vortex theory has a phase for $\lambda \gg 1$, $\lambda_{m} \ll 1$ in which both fields $c^{(1)}$ and $c^{(2)}$ are deconfined while the monopole field $\theta^{(m)}$ is gapped. The screened vortex theory has two photons in this phase. Including fluctuations in the rank-2 field $\mathfrak{g}^{-}$, it obtains a mass and "eats" in the process one photon, so there remains precisely one photon $\left[\nabla \times\left(c^{(1)}+c^{(2)}\right)\right]^{2}$. In the physical boson model, this is the fractionalized Coulomb phase at the focus of this paper.

On the other hand, in the regime $\lambda \gg 1, \lambda_{m} \gg 1$ monopoles are also proliferated and destroy the photon, so this becomes the conventional Mott insulator of bosons.

Finally, for small $\lambda \ll 1$ both dual gauge fields $c^{(1)}$ and $c^{(2)}$ are confined. The dual electric field lines which are the vortices of the physical boson model are gapped, and this corresponds to the superfluid phase of bosons. As we vary the parameter $\lambda_{m}$ inside this phase, there is a transition in the properties of these line excitations from a unique core state to two degenerate core states.

The dual formulation reveals some details of this core transition. Specifically, we consider a single vortex in the screened vortex theory-this allows us to avoid complications of coupling the vortex dynamics to phonons and focus on the core physics.

It is convenient to use the Hamiltonian formulation

$$
\begin{aligned}
H_{\text {screened }}= & u_{m} \sum n_{R}^{2}-t_{m} \sum \cos \left(\nabla \theta^{(m)}-\boldsymbol{c}^{(1)}-\boldsymbol{c}^{(2)}\right) \\
& +u_{v} \sum\left(\boldsymbol{e}_{1}^{2}+\boldsymbol{e}_{2}^{2}\right)-t_{v} \sum\left[\cos \left(\nabla \times \boldsymbol{c}^{(1)}\right)\right. \\
& \left.+\cos \left(\nabla \times \boldsymbol{c}^{(2)}\right)\right],
\end{aligned}
$$

with the Hilbert space constraints

$$
\boldsymbol{\nabla} \cdot \boldsymbol{e}_{1}=\boldsymbol{\nabla} \cdot \boldsymbol{e}_{2}=n_{R}
$$

Here $u_{m}$ and $t_{m}$ are the bare monopole gap and hopping amplitude, while $u_{v}$ is bare vortex core energy per unit length and $t_{v}$ is vortex hopping amplitude. The superfluid phase of the boson model corresponds to the the confining phase $u_{v}$ $\gg t_{v}$ of $H_{\text {screened. }}$. The electric field $\boldsymbol{e} \equiv \boldsymbol{e}_{1}-\boldsymbol{e}_{2}$ is the conserved physical vorticity, and we study a strength-1 line of $e$ oriented in say, the $\hat{x}$ direction. In the limit $u_{v} \gg t_{v}$, we have a straight line $e_{x}=1$, but its segments can be realized as either $\left(e_{1 x}, e_{2 x}\right)=(1,0)$ or $(0,-1)$. Each juncture $(0,-1) \rightarrow(1,0)$ has a +1 monopole and $(1,0) \rightarrow(0,-1)$ has a -1 monopole, and the quantum dynamics is determined by the competition between the $u_{m}$ and $t_{m}$ terms. Writing $\sigma_{l}^{z}=+1$ for $(1,0)$ segment and $\sigma_{l}^{z}=-1$ for $(0,-1)$ segment, we obtain the following 1D Hamiltonian for the straight line:

$$
H_{\text {core }}=-\frac{u_{m}}{2} \sum_{l} \hat{\sigma}_{l}^{z} \hat{\sigma}_{l+1}^{z}-\frac{t_{m}}{2} \sum_{l} \hat{\sigma}_{l}^{x} .
$$

This is simply the one-dimensional quantum Ising model and has two phases: For $u_{m}<t_{m}$, we obtain a paramagnet in $\sigma^{z}$ and the ground state is unique, schematically $\sigma^{x}=1$. In this case, the monopoles proliferate along the line, and the $e=1$ line is unique. On the other hand, for $u_{m}>t_{m}$, we obtain a ferromagnet with two degenerate ground states $\sigma^{z}=+1$ and $\sigma^{z}=-1$. The monopoles remain gapped along the line, and we have two distinct $e=1$ lines. The core Hamiltonian can be also written in dual Ising variables, and this gives a description in terms of domain wall particles which we identify with the monopoles.

The above analysis is valid in the limit $u_{v} \gg t_{v}$, but we expect the two regimes and the transition between them to extend throughout the confined phase of $H_{\text {screened. }}$. In the superfluid phase of the original boson model, we conjecture that the corresponding vortex core transition line extends all the way to the insulating phases as shown in Fig. 5. 


\section{APPENDIX B: DUALITY ANALYSIS FOR BOSONS AT HALF-INTEGER FILLING}

Here we generalize the analysis in Sec. IV A to bosons at half-integer filling and study the resulting dual theory directly. At half-filling, we simply make the replacement

$$
j_{i \tau}^{2} \rightarrow\left(j_{i \tau}-\frac{1}{2}\right)^{2}
$$

in the final expression, Eq. (27), while the rest remains unchanged. As in $(2+1) \mathrm{D}$, the noninteger boson density enters as an external "field" seen by the vortices, except that vortices are now lines and the gauge potential is a rank-2 tensor.

A convenient formulation is obtained by finding static $\mathfrak{g}_{\rho \sigma}^{0}$ such that

$$
\frac{1}{2} \delta_{\mu \tau}=\frac{1}{2} \epsilon_{\mu \nu \rho \sigma} \nabla_{\nu} \frac{\mathfrak{g}_{\rho \sigma}^{0}}{2 \pi} .
$$

If $\mathfrak{g}_{\rho \sigma}^{0}$ are viewed as fluxes through the dual lattice plackets, $\mathfrak{g}^{0}$ has total flux of $\pi$ coming out of each spatial cube. Shifting $\mathfrak{g}$ by $\mathfrak{g}^{0}$ we get

$$
\begin{aligned}
Z= & \int_{-\infty}^{\infty}\left[D \mathfrak{g}_{I \rho \sigma}\right]^{\prime} \int_{-\pi}^{\pi}\left[D c_{I \rho}\right] \exp \left[-\frac{1}{2 \beta} \sum(d \mathfrak{g})^{2}\right] \\
& \times \exp \left[\lambda \sum_{I, \rho<\sigma} \cos \left(\nabla_{\rho} c_{\sigma}-\nabla_{\sigma} c_{\rho}-\mathfrak{g}_{I \rho \sigma}-\mathfrak{g}_{I \rho \sigma}^{0}\right)\right] .
\end{aligned}
$$

Thus, there is a static contribution to the rank- 2 gauge potential corresponding to placing half of a magnetic monopole inside each spatial cube. When a vortex line evolves in space-time, this gives a Berry phase contribution of $\pi$ for each cube in the 3D volume swept by the line.

As in our discussion for bosons at integer filling, it is helpful to first consider the compact $U(1)$ part with only the static $\mathfrak{g}_{\rho \sigma}^{0}$. This "frustrated" lattice gauge theory has not been studied to our knowledge. At present, we do not have direct tools for approaching this problem similar to the ones used for frustrated systems with global symmetries. We can still describe what we expect to happen in such a model.

Two phases of the frustrated lattice gauge theory are clearly identified. For small $\lambda$, we expect confinement in $e^{i c_{I \rho}}$, which corresponds (upon restoring fluctuations in $\mathfrak{g}_{\rho \sigma}$ ) to the superfluid phase of the bosons.

For large $\lambda$, we expect deconfinement in $e^{i c_{I \rho}}$, but we need to be more specific since there can be different patterns of deconfinement in the presence of $\mathfrak{g}_{\rho \sigma}^{0}$. For the hard-spin action written above, we can proceed by considering first the classical ground states and obtain the following picture. For each spatial cube, the total outgoing flux of $e^{i\left(\nabla_{\rho} c_{\sigma}-\nabla_{\sigma} c_{\rho}-\mathfrak{g}_{\rho \sigma}^{0}\right)}$ must be $\pi$ modulo $2 \pi$ because of the compactness of the gauge field. For an individual cube, the lowest energy is obtained by dividing this flux equally among the six faces. On the lattice, we put such cubes with the outgoing placket fluxes of $+\pi / 6$ and cubes with the outgoing fluxes of $-\pi / 6$ in a checkerboard pattern, and there are two degenerate ground states. This structure survives as a phase in the frustrated lattice gauge theory. For the original bosonic system, this phase corresponds to checkerboard charge density wave.
Indeed, when we allow fluctuations in $\mathfrak{g}_{\rho \sigma}$, the above staggered character is imprinted on the fluxes $g_{\rho \sigma}$, thus producing staggered boson density. The two states correspond to two ways to register this charge density wave (CDW) on the lattice.

Other deconfinement patterns are also possible, but likely require some deformation of the above simple action to be stabilized. For example, it is possible to have a deconfined state with an energy density wave, and this would correspond to some VBS phase for the original bosons.

Of direct interest here is the possibility of deconfinement in $e^{i c_{I \rho}}$ that produces $t w o$ photons in the low-energy description and does not break any lattice symmetry. Allowing fluctuations in $\mathfrak{g}_{\rho \sigma}$, only one photon remains, and this would give the sought for fractionalized Coulomb phase at half-integer filling. We know that this can indeed happen in the original bosonic system, and Appendixes A and $\mathrm{C}$ provide an indirect route to describe such a phase. It would be interesting to explore this in the above frustrated lattice gauge theory in more detail and develop direct analytical tools for treating such theories.

\section{APPENDIX C: DUALITY FOR TWO-CHARGON THEORY AT HALF-INTEGER FILLING}

In this appendix, we derive a dual description for a twochargon gauge theory corresponding to bosons at half-integer filling. Our analysis is a direct extension to $(3+1) \mathrm{D}$ of Appendix E of Ref. 15, which should be consulted for further details. Following Ref. 15, we arrive at a path integral which has the same form as at integer filling Eq. (A1) but with replacements

$$
\left(j_{1 i \tau}\right)^{2} \rightarrow\left(j_{1 i \tau}-n_{0} \eta_{i}\right)^{2}, \quad\left(j_{2 i \tau}\right)^{2} \rightarrow\left(j_{2 i \tau}-n_{0} \eta_{i}\right)^{2},
$$

and an additional term in the action

$$
S_{B}=i \sum_{i} \eta_{i} a_{i \tau}
$$

In the limit $K \rightarrow 0$ (i.e., in the absence of the gauge field dynamics), any choice of the "chemical potential" $n_{0}$ for the on-site gauge charge will give the same result due to the constraint $j_{1 i \tau}+j_{2 i \tau}=$ const. This is no longer true for finite $K$, but the qualitative behavior is expected to be insensitive to $n_{0}$, and we will use this freedom to make the structure of the dual theory more transparent.

We proceed as in Appendix A while treating the phase term $S_{B}$ as in our analysis of the monopole Berry phase for spin-1/2 systems in Sec. VI. Specifically, we take $f_{\mu \nu}^{0}$ defined by conditions in Eqs. (38) and (39) and make the corresponding change of variables $f_{\mu \nu}=\tilde{f}_{\mu \nu}+f_{\mu \nu}^{0}$. In terms of the variables $j_{1 \mu}, j_{2 \mu}, \tilde{f}_{\mu \nu}$, we obtain the same action as at integer filling except for additional contributions

$$
\frac{1}{K} \sum_{\mu<\nu} \tilde{f}_{\mu \nu} f_{\mu \nu}^{0}-\frac{n_{0}}{\beta} \sum \eta_{i} j_{i \tau}^{+}+i \sum_{\mu<\nu} f_{\mu \nu}^{0} 2 \pi u_{\mu \nu} .
$$

In particular, upon integrating out $\phi_{1}, \phi_{2}, a_{\mu}$, we have $j_{\mu}^{+}$ $=\nabla_{\nu} \tilde{f}_{\mu \nu}$. The first two terms cancel each other if $K n_{0} / \beta$ 


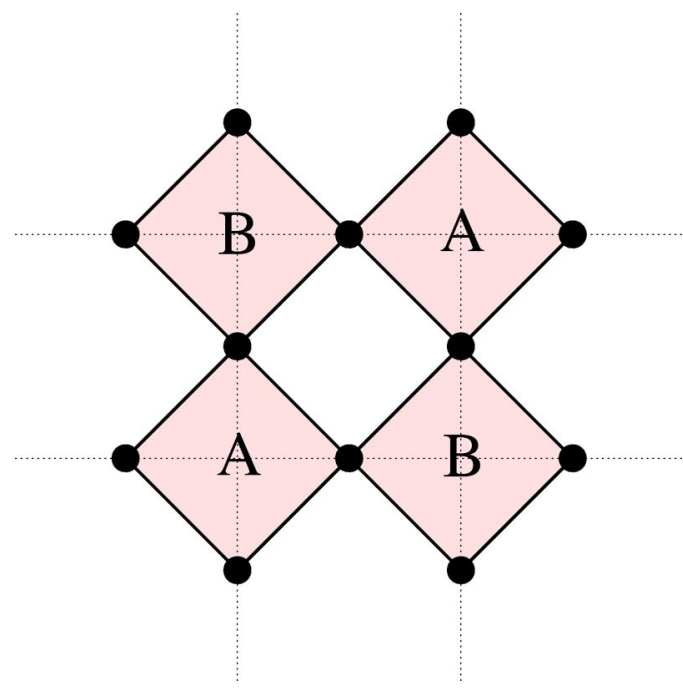

FIG. 8. Quantum Ising model that realizes $P^{*}$ phase in two dimensions. Spins are located on the link-centered sites of a square lattice. Each shaded diamond indicates the $U$ term in the Hamiltonian, Eq. (D1).

$=1 / 12$ [see Eq. (42)]. The importance of the specific form for $f_{\mu \nu}^{0}$ is that for general $n_{0}$ the first two terms add up to $\left(\delta n_{0} / \beta\right) \Sigma_{i} \eta_{i} j_{i \tau}^{+}$, which can be viewed as a staggered chemical potential seen by the gauge charges. As long as this potential is small, it effectively averages out and does not modify the low-energy modes; in the following, we set $\delta n_{0}=0$.

The remaining phase term can be expressed entirely in terms of the monopole currents $J_{\rho}^{(m)}$,

$$
\tilde{S}_{B}=i \sum J_{\rho}^{(m)} X_{\rho}^{0},
$$

with $X_{\rho}^{0}$ defined in Sec. VI. This term needs to be added to the dual action expressed in terms of the topological defects, Eq. (A10). Thus, we have managed to absorb all effects of half-integer filling into the monopole Berry phases. To obtain the final action in terms of the dual fields $\theta^{(m)}, c^{(1)}, c^{(2)}$, we simply take the expression at integer filling, Eq. (A12), and make the replacement

$$
\nabla_{\rho} \theta^{(m)} \rightarrow \nabla_{\rho} \theta^{(m)}-X_{\rho}^{0}
$$

\section{APPENDIX D: ISING $P^{*}$ PHASE IN $(2+1)$ D}

Toy model. Here we consider a toy model which is a $2 \mathrm{D}$ quantum Ising version of the 3D boson model of Sec. II. As we will see, some physical aspects are similar in the two problems, while the formalism is much simpler in the Ising case and gives some guidance for the bosons. Ising spins reside on links of a square lattice, and the Hamiltonian is

$$
H=-J \sum_{\left\langle l l^{\prime}\right\rangle} \sigma_{l}^{z} \sigma_{l^{\prime}}^{z}-\Gamma \sum_{l} \sigma_{l}^{x}-U \sum_{r} \prod_{l \in r} \sigma_{l}^{x} .
$$

The lattice is shown in Fig. 8. The spins can be viewed as forming a network of corner-sharing diamonds, and we a introduced four-spin interaction $U$ on each diamond. For simplicity, we take $U>0$.
The phase diagram of the model can be analyzed as in Sec. II. When the Ising coupling dominates, $J \gg \Gamma, U$, the system is ferromagnetically ordered. When the transverse fields dominate, $\Gamma, U \gg J$, the system is a conventional Ising paramagnet.

The intermediate regime $U \gg J \gg \sqrt{\Gamma U}$ is most interesting and realizes an unusual topologically ordered paramagnet (Ising $P^{*}$ phase). The excitation spectrum in this phase contains a gapped Ising vortex (vison) and gapped Ising matter fields. The argument is similar for the Ising and boson models. In the large- $U$ limit, the ground-state sector is determined by the projection $\Pi_{l \in r} \sigma_{l}^{x}=1$ for all $r$, and the effective Hamiltonian in this sector is

$$
H_{\mathrm{eff}}^{(0)}=-\Gamma \sum_{l} \sigma_{l}^{x}-K \sum_{\square} \sigma_{12}^{z} \sigma_{23}^{z} \sigma_{34}^{z} \sigma_{41}^{z}
$$

with $K=J^{2} / U$. This is just the familiar Ising gauge theory. For $K \lesssim \Gamma$ the theory is confining and corresponds to the conventional paramagnet, but for $K \gtrsim \Gamma$ we have a deconfined phase and this is the Ising $P^{*}$ phase.

The topology of the phase diagram is the same as in Fig. 4 , with the correspondences superfluid $\rightarrow$ ferromagnet, conventional insulator $\rightarrow$ conventional paramagnet, and fractionalized insulator $\rightarrow P^{*}$ paramagnet. We are led to ask similar questions on the relationships among the phases, in particular, how to view the $P^{*}$ phase coming from the ferromagnet. In the ferromagnet, excitations are domain walls, and we can think of a paramagnet as a result of proliferating these. The $P^{*}$ phase features the vison, which is a topological point excitation, and we ask how this appears in the domain wall condensate.

To push the analogy somewhat further, in $(3+1) \mathrm{D}$ the appearance of the Coulomb phase with the monopole excitation is "natural" when an $O(3)$ spin model is disordered without proliferating hedgehogs (gapped hedgehogs then become the monopoles). As discussed in the main text, the appearance of the Coulomb phase in a nominally $O(2)$ model is more subtle in terms of the vortex line defects of the ordered phase. Similar situation occurs in $(2+1) \mathrm{D}$, where the appearance of the $Z_{2}$ fractionalized phase with the vison excitation is natural when an $O(2)$ model is disordered by proliferating double vortices but not single vortices, but the appearance of such $Z_{2}$ phase is not so clear in a nominally Ising model. We argue that the key to this puzzle lies in the presence of two degenerate but distinct domain walls in the ferromagnetic phase near the $P^{*}$ phase.

Direct analysis of domain walls. In the present model, we can perform a direct analysis in the limit of small $\Gamma$, i.e., in the upper right corner of the phase diagram Fig. 4.

When $\Gamma=0$ in the microscopic model, we find that for each square lattice placket the corresponding $Z_{2}$ "flux" $\Phi_{P}$ $\equiv \sigma_{12}^{z} \sigma_{23}^{z} \sigma_{34}^{z} \sigma_{41}^{z}$ is conserved. This simplifies the analysis, since in each sector with fixed $\Phi$ 's the Hamiltonian decouples into two quantum Ising models. Indeed, let us work in the $\sigma^{z}$ basis and fix a sector $\left\{\Phi_{P}\right\}$. Let us find a specific realization $\left\{\sigma_{r r^{\prime}}^{z(0)}\right\}$ of the fluxes, $\Phi_{P}=\sigma_{12}^{z(0)} \sigma_{23}^{z(0)} \sigma_{34}^{z(0)} \sigma_{41}^{z(0)} \cdot\left\{\sigma_{r r^{\prime}}^{z}\right\}$ belongs to this sector if we can write $\sigma_{r r^{\prime}}^{z}=S_{r^{\prime}}^{z} S_{r^{\prime}}^{z} \sigma_{r r^{\prime}}^{z(0)}$, and we 
can label all states in the sector by the new Ising variables $\left\{S_{r}^{z}\right\}$. The action of the Hamiltonian in this sector reads

$$
H_{\Phi}=-\sum_{\left\langle A A^{\prime}\right\rangle} J_{A A^{\prime}} S_{A}^{z} S_{A^{\prime}}^{z}-U \sum_{A} S_{A}^{x}-\sum_{\left\langle B B^{\prime}\right\rangle} J_{B B^{\prime}} S_{B^{z}}^{z} S_{B^{\prime}}^{z}-U \sum_{B} S_{B}^{x} .
$$

Here $A$ and $B$ refer to the two sublattices of the square lattice. Ising couplings $J_{A A^{\prime}}$ and $J_{B B^{\prime}}$ depend on the sector $\left\{\Phi_{P}\right\}$; for example, $J_{A A^{\prime}}$ is determined by considering the square lattice placket $\left[1_{A}, 2,3_{A^{\prime}}, 4\right]$ that has $A A^{\prime}$ as its diagonal: $J_{A A^{\prime}}=J\left(\sigma_{12}^{z(0)} \sigma_{23}^{z(0)}+\sigma_{14}^{z(0)} \sigma_{43}^{z(0)}\right)$.

For arbitrary $J / U$ (with $\Gamma=0$ ) the ground-state sector has no fluxes, $\Phi_{P}=1$, and $J_{A A^{\prime}}=J_{B B^{\prime}}=2 J$. As we change $J / U$, we have a simultaneous Ising ordering transition for spins $S_{A}$ and $S_{B}$. The gap to the nearest sector is at least of order $J$ in the ferromagnetic phase and of order $J^{2} / U$ in the spindisordered phase.

Consider the ferromagnetic phase. In the sector with no fluxes, excitations are domain walls for the Ising variables $S_{A}$ or $S_{B}$. When translated to the original variables $\sigma_{r r^{\prime}}^{z}$, a domain wall in $S_{A}$ is a physical domain wall that passes through the $B$ sublattice sites, while a domain wall in $S_{B}$ is a physical domain wall that passes through the $A$ sites. In a sector with two visons, one finds that the lowest-energy state has an $A$-type domain wall (defined by frustrated bonds $J_{A A^{\prime}} S_{A^{z}}^{z} S_{A^{\prime}}^{z}$ ) connecting the two visons and also a $B$-type domain wall connecting the visons. Thus, visons act as sources for both domain walls and are linearly confined.

Consider now the spin-disordered phase. It can be viewed as a condensate of both domain walls, and has two Ising matter fields $S_{A}$ and $S_{B}$ with the mass of order $U$. The different sectors are accounted for as vison excitations with the vison gap of order $J^{2} / U$.

For nonzero $\Gamma$, the different sectors will mix, but the above description of the phases will remain true as long as $\Gamma$ is much smaller than the gap to the nearest sector. Small nonzero $\Gamma$ will induce energy-energy coupling between the two Ising models; this will affect the nature of the transition, either making it first-order or driving to a different universality class; however, the above physical picture with two distinct domain walls remains.

A phenomenological gauge theory description similar to the two-chargon theory is also possible. Again, it relies on the observation that in the $P^{*}$ phase we find two distinct Ising matter excitations. The phenomenological Hamiltonian is

$$
\begin{aligned}
H_{2 \mathrm{is}}= & -U \sum_{r}\left(\tau_{1 r}^{x}+\tau_{2 r}^{x}\right)-J \sum_{\left\langle r r^{\prime}\right\rangle}\left(\sigma_{r r^{\prime}}^{z} \tau_{1 r_{1 r^{\prime}}^{z}}^{z}+\sigma_{r r^{\prime}}^{z} \tau_{2 r}^{z} \tau_{2 r^{\prime}}^{z}\right) \\
& -\Gamma \sum_{\left\langle r r^{\prime}\right\rangle} \sigma_{r r^{\prime}}^{x}-K \sum_{\square} \sigma_{12}^{z} \sigma_{23}^{z} \sigma_{34}^{z} \sigma_{41}^{z} ;
\end{aligned}
$$

it contains two Ising matter fields $\tau_{1 r}, \tau_{2 r}$ coupled to an Ising gauge field $\sigma_{r r}$. The Hilbert space of the theory is defined by

$$
\tau_{1 r}^{x} \tau_{2 r}^{x} \prod_{r^{\prime} \in r} \sigma_{r r^{\prime}}^{x}=1 .
$$

We emphasize that this model is not derived from the Hamiltonian, Eq. (D1) (in particular, the reader should not be con- fused by our reuse of the letters for the coupling constants). Rather, this model is to be considered as capturing the relevant physics of the $P^{*}$ phase.

The analysis of the phenomenological gauge theory proceeds exactly as in the two-chargon theory, and the phase diagram has the same topology as in Fig. 5. The ordered phase has ferromagnetic order in the "physical" spin $\tau_{r}^{z}$ $\equiv \tau_{1 r}^{z} \tau_{2 r}^{z}$. In the ferromagnetic phase, we have domain wall excitations, and a domain wall segment crossing a link $\tau_{r}^{z} \tau_{r^{\prime}}^{z}=-1$ can be realized as either a domain wall in $\tau_{1}$ $\left(\sigma_{r r^{\prime}}^{z} \tau_{1 r}^{z} \tau_{1 r^{\prime}}^{z}=-1\right)$ or a domain wall in $\tau_{2}$. $Z_{2}$ flux (vison) acts as a source for both domain walls, and there is a linearly confining potential between a pair of visons in the ferromagnetic phase. On a physical domain wall, however, the vison costs only finite energy and can hop along the domain wall; this hopping represents quantum tunneling between the two microscopic realizations of the hopped segment. The vison can be thought of as a point "domain wall" for the order along the line which is the Ising domain wall of the ferromagnetic phase.

We expect two possibilities: One is that visons proliferate along the domain wall, in which case the domain wall is unique. The other possibility is that visons remain gapped inside the domain wall, and there are two distinct domain walls. A precise formulation is to consider an externally imposed domain wall in the ferromagnetic phase. As we change the parameters in the ferromagnetic phase, there is a transition from a disordered domain wall with a unique ground state to an ordered domain wall with two degenerate ground states, and this domain wall ordering transition is of (1 $+1)$ D Ising type. When we have ordered domain walls and proliferate both with equal amplitudes, the $P^{*}$ phase results.

The mathematical formalism for the above picture is transformation to dual variables, which is readily done in the Hamiltonian language. The dual Hamiltonian is

$$
\begin{aligned}
H_{2 \mathrm{is}, \text { dual }}= & -U \sum_{\square}\left(C_{12}^{z} C_{23}^{z} C_{34}^{z} C_{41}^{z}+D_{12}^{z} D_{23}^{z} D_{34}^{z} D_{41}^{z}\right) \\
& -J \sum_{\left\langle R R^{\prime}\right\rangle}\left(C_{R R^{\prime}}^{x}+D_{R R^{\prime}}^{x}\right)-\Gamma \sum_{\left\langle R R^{\prime}\right\rangle} V_{R}^{z} V_{R^{\prime}}^{z} C_{R R^{\prime}}^{z} D_{R R^{\prime}}^{z} \\
& -K \sum_{R} V_{R}^{x} ;
\end{aligned}
$$

the Hilbert space is defined by

$$
V_{R}^{x} \prod_{R^{\prime} \in R} C_{R R^{\prime}}^{x}=1, \quad V_{R}^{x} \prod_{R^{\prime} \in R} D_{R R^{\prime}}^{x}=1 .
$$

The two domain wall variables are denoted as $C, D$, while the $Z_{2}$ flux variable is denoted as $V: C_{R R^{\prime}}^{x} \equiv \sigma_{r r^{\prime}}^{z} \tau_{1 r}^{z} \tau_{1 r^{\prime}}^{z}$, $D_{R R^{\prime}}^{x} \equiv \sigma_{r r^{\prime}}^{z} \tau_{2 r^{\prime}}^{z} \tau_{2 r^{\prime}}^{z}$, and $V_{R}^{x} \equiv \sigma_{12}^{z} \sigma_{23}^{z} \sigma_{34}^{z} \sigma_{41}^{z}$. Compare this with the dual formulation, Eq. (A12), of the two-chargon theory. The ferromagnetic phase in the dual language corresponds to confinement in both dual gauge fields $C$ and $D$. In the $P^{*}$ phase, both these fields become deconfined while the dual matter field $V$ where the two connect remains gapped. 
${ }^{1}$ N. Read and S. Sachdev, Phys. Rev. Lett. 66, 1773 (1991); X.-G. Wen, Phys. Rev. B 44, 2664 (1991).

${ }^{2}$ R. Moessner and S. L. Sondhi, Phys. Rev. Lett. 86, 1881 (2001).

${ }^{3}$ L. B. Ioffe et al., Nature (London) 415, 503 (2002).

${ }^{4}$ L. Balents, M. P. A. Fisher, and S. M. Girvin, Phys. Rev. B 65 , 224412 (2002).

${ }^{5}$ O. I. Motrunich and T. Senthil, Phys. Rev. Lett. 89, 277004 (2002).

${ }^{6}$ X.-G. Wen, Phys. Rev. Lett. 88, 011602 (2002); Phys. Rev. B 68, 115413 (2003).

${ }^{7}$ D. A. Huse, W. Krauth, R. Moessner, and S. L. Sondhi, Phys. Rev. Lett. 91, 167004 (2003).

${ }^{8}$ M. Hermele, M. P. A. Fisher, and L. Balents, Phys. Rev. B 69, 064404 (2004).

${ }^{9}$ R. Moessner and S. L. Sondhi, Phys. Rev. B 68, 184512 (2003).

${ }^{10}$ D. Foerster, H. B. Nielsen, and M. Ninomiya, Phys. Lett. 94, 135 (1980).

${ }^{11}$ M. P. A. Fisher and D. H. Lee, Phys. Rev. B 39, 2756 (1989).

${ }^{12}$ L. Balents, M. P. A. Fisher, and C. Nayak, Phys. Rev. B 60, 1654 (1999); 61, 6307 (2000).

${ }^{13}$ T. Senthil and M. P. A. Fisher, Phys. Rev. B 62, 7850 (2000).
${ }^{14}$ C. Lannert, M. P. A. Fisher, and T. Senthil, Phys. Rev. B 63, 134510 (2001).

${ }^{15}$ T. Senthil, A. Vishwanath, L. Balents, S. Sachdev, and M. P. A. Fisher, Science 303, 1490 (2004); T. Senthil, L. Balents, S. Sachdev, A. Vishwanath, and M. P. A. Fisher, Phys. Rev. B 70, 144407 (2004).

${ }^{16}$ E. Fradkin and S. H. Shenker, Phys. Rev. D 19, 3682 (1979).

${ }^{17}$ B. I. Halperin, T. C. Lubensky, and S.-k. Ma, Phys. Rev. Lett. 32, 292 (1974)

${ }^{18}$ A. M. Polyakov, Gauge Fields and Strings (Harwood Academic, New York, 1987).

${ }^{19}$ M. Peskin, Ann. Phys. (N.Y.) 113, 122 (1978); R. Savit, Rev. Mod. Phys. 52, 453 (1980).

${ }^{20}$ C. Dasgupta and B. I. Halperin, Phys. Rev. Lett. 47, 1556 (1981).

${ }^{21}$ M. P. A. Fisher and O. I. Motrunich (unpublished).

${ }^{22}$ F. D. M. Haldane, Phys. Rev. Lett. 61, 1029 (1988).

${ }^{23}$ N. Read and S. Sachdev, Phys. Rev. Lett. 62, 1694 (1989).

${ }^{24}$ S. Sachdev and R. Jalabert, Mod. Phys. Lett. B 4, 1043 (1990).

${ }^{25}$ S. Sachdev and K. Park, Ann. Phys. (N.Y.) 298, 58 (2002).

${ }^{26}$ M. Levin and T. Senthil, Phys. Rev. B 70, 220403 (2004). 